\title{
GENERIC UNIQUENESS OF MINIMAL CONFIGURATIONS WITH RATIONAL ROTATION NUMBERS IN AUBRY-MATHER THEORY
}

\author{
ALEXANDER J. ZASLAVSKI
}

Received 18 November 2002

We study $(h)$-minimal configurations in Aubry-Mather theory, where $h$ belongs to a complete metric space of functions. Such minimal configurations have definite rotation number. We establish the existence of a set of functions, which is a countable intersection of open everywhere dense subsets of the space and such that for each element $h$ of this set and each rational number $\alpha$, the following properties hold: (i) there exist three different (h)-minimal configurations with rotation number $\alpha$; (ii) any (h)-minimal configuration with rotation number $\alpha$ is a translation of one of these configurations.

\section{Introduction}

Let $\mathbb{Z}$ be a set of all integers. A configuration is a bi-infinite sequence $x=\left(x_{i}\right)_{i \in \mathbb{Z}}$ $\in \mathbb{R}^{\mathbb{Z}}$. The set $\mathbb{R}^{\mathbb{Z}}$ will be endowed with the product topology and the partial order defined by $x<y$ if and only if $x_{i}<y_{i}$ for all $i \in \mathbb{Z}$.

We have an order-preserving action $T: \mathbb{Z}^{2} \times \mathbb{R}^{\mathbb{Z}} \rightarrow \mathbb{R}^{\mathbb{Z}}$ defined by

$$
\begin{gathered}
T(k, x)=T_{k} x=y \Longleftrightarrow k=\left(k_{1}, k_{2}\right) \in \mathbb{Z}^{2}, \\
x, y \in \mathbb{R}^{\mathbb{Z}}, \quad y_{i}=x_{i-k_{1}}+k_{2} \quad \forall i \in \mathbb{Z} .
\end{gathered}
$$

Let $x, y \in \mathbb{R}^{\mathbb{Z}}$. We say that $y$ is a translation of $x$ if there is $n=\left(n_{1}, n_{2}\right) \in \mathbb{Z}^{2}$ such that $y=T_{n} x$.

Let $h: \mathbb{R}^{2} \rightarrow \mathbb{R}^{1}$ be a continuous function. We extend $h$ to arbitrary finite segments $\left(x_{j}, \ldots, x_{k}\right), j<k$, of configurations $x \in \mathbb{R}^{\mathbb{Z}}$ by

$$
h\left(x_{j}, \ldots, x_{k}\right):=\sum_{i=j}^{k-1} h\left(x_{i}, x_{i+1}\right) .
$$

A segment $\left(x_{j}, \ldots, x_{k}\right)$ is called $(h)$-minimal if $h\left(x_{j}, \ldots, x_{k}\right) \leq h\left(y_{j}, \ldots, y_{k}\right)$ whenever $x_{j}=y_{j}$ and $x_{k}=y_{k}$. 
We assume that $h$ has the following properties [3, 4]:

(H1) for all $(\xi, \eta) \in \mathbb{R}^{2}, h(\xi+1, \eta+1)=h(\xi, \eta)$;

(H2) $\lim _{|\eta| \rightarrow \infty} h(\xi, \xi+\eta)=\infty$ uniformly in $\xi$;

(H3) if $\xi_{1}<\xi_{2}$ and $\eta_{1}<\eta_{2}$, then

$$
h\left(\xi_{1}, \eta_{1}\right)+h\left(\xi_{2}, \eta_{2}\right)<h\left(\xi_{1}, \eta_{2}\right)+h\left(\xi_{2}, \eta_{1}\right) ;
$$

(H4) if $\left(x_{-1}, x_{0}, x_{1}\right) \neq\left(y_{-1}, y_{0}, y_{1}\right)$ are $(h)$-minimal segments and $x_{0}=y_{0}$, then

$$
\left(x_{-1}-y_{-1}\right)\left(x_{1}-y_{1}\right)<0 \text {. }
$$

A configuration $x \in \mathbb{R}^{\mathbb{Z}}$ is $(h)$-minimal if, for each pair of integers $j$ and $k$ satisfying $j<k$ and each finite segment $\left\{y_{i}\right\}_{i=j}^{k} \subset \mathbb{R}^{1}$ satisfying $y_{j}=x_{j}$ and $y_{k}=x_{k}$, the inequality $h\left(x_{j}, \ldots, x_{k}\right) \leq h\left(y_{j}, \ldots, y_{k}\right)$ holds. Denote by $M(h)$ the set of all $(h)$-minimal configurations. It is known that the set $M(h)$ is closed [2, 3].

The notion of global minimizers $((h)$-minimal configurations in the present paper) is crucial to the Aubry-Mather theory. The works by Aubry and Mather were begun independently and with different motivations but led to similar results by different methods. While Mather [12] studied area-preserving annulus mappings as they occur as section mappings for Hamiltonian systems of two degrees of freedom, Aubry [1] investigated certain models of solid state physics related to dislocations in one-dimensional crystals. For more details on Aubry-Mather theory, see [1, 2, 3, 4, 12, 13, 14, 15, 18, 19]. For the usage of the notion of global minimizers in the related topics of calculus of variations, partial differential equations, and geometry, see also $[3,4,5,6,7,8,10,11,16,17,20,21]$.

We briefly review the definitions, notions, and some basic results from Aubry-Mather theory $[2,3]$.

Definition 1.1. The configurations $x \in \mathbb{R}^{\mathbb{Z}}$ and $x^{*} \in \mathbb{R}^{\mathbb{Z}}$ cross

(a) at $i \in \mathbb{Z}$ if $x_{i}=x_{i}^{*}$ and $\left(x_{i-1}-x_{i-1}^{*}\right)\left(x_{i+1}-x_{i+1}^{*}\right)<0$,

(b) between $i$ and $i+1$ if $\left(x_{i}-x_{i}^{*}\right)\left(x_{i+1}-x_{i+1}^{*}\right)<0$.

Definition 1.2. The configuration $x \in \mathbb{R}^{\mathbb{Z}}$ is periodic with period $(q, p) \in(\mathbb{Z} \backslash\{0\}) \times \mathbb{Z}$ if $T_{(q, p)} x=x$.

Remark 1.3. Assume that $h=h\left(\xi_{1}, \xi_{2}\right) \in \mathbb{C}^{2}\left(\mathbb{R}^{2}\right)$ and $\left(\partial^{2} h / \partial \xi_{1} \partial \xi_{2}\right)(u, v)<0$ for all $(u, v) \in$ $\mathbb{R}^{2}$. It is not difficult to show that (H3) and (H4) hold. Moreover, we can show that if $h \in \mathbb{C}^{2}\left(\mathbb{R}^{2}\right)$, then (H3) holds if and only if

$$
\left\{(u, v) \in \mathbb{R}^{2}:\left(\frac{\partial^{2} h}{\partial \xi_{1} \partial \xi_{2}}\right)(u, v)<0\right\}
$$

is an everywhere dense subset of $\mathbb{R}^{2}$.

We have the following result [3, Corollary 3.16, Theorem 3.17]. 
Proposition 1.4. There exists a continuous function $\alpha^{(h)}: M(h) \rightarrow \mathbb{R}^{1}$ with the following properties:

(i) for all $x \in \mathcal{M}(h), i \in \mathbb{Z}$,

$$
\left|x_{i}-x_{0}-i \alpha^{(h)}(x)\right|<1
$$

(ii) if $x \in M(h)$ is periodic with period $(q, p) \in \mathbb{Z}^{2}$, then $\alpha^{(h)}(x)=p / q$;

(iii) for all $\alpha \in \mathbb{R}^{1}$, the set $\left\{x \in \mathcal{M}(h): \alpha^{(h)}(x)=\alpha\right\} \neq \varnothing$.

Remark 1.5. We call $\alpha^{(h)}(x)$ the rotation number of $x \in \mathcal{M}(h)$.

For each $\alpha \in \mathbb{R}^{1}$, define

$$
M(h, \alpha)=\left\{x \in \mathcal{M}(h): \alpha^{(h)}(x)=\alpha\right\} .
$$

We study $\mathcal{M}(h, \alpha)$ with rational $\alpha \in \mathbb{R}^{1}$.

Let a rational $\alpha=p / q$ be an irreducible fraction, where $q \geq 1$ and $p$ are integers. Denote by $\mathcal{M}^{\text {per }}(h, \alpha)$ the set of all periodic $(h)$-minimal configurations $x \in \mathcal{M}(h, \alpha)$ which satisfy $T_{(q, p)} x=x$, equivalently, $x_{i-q}+p=x_{i}$, for all $i \in \mathbb{Z}$.

For the proof of the following result, see $[2,3]$.

Proposition 1.6. $M^{\mathrm{per}}(h, \alpha)$ is a nonempty closed totally ordered set. Moreover, if $x \in$ $M^{\mathrm{per}}(h, \alpha)$, then $x$ is a minimizer of $h_{q p}: P_{q p} \rightarrow \mathbb{R}^{1}$, where

$$
h_{q p}(x)=h\left(x_{0}, \ldots, x_{q}\right), \quad P_{q p}=\left\{x \in \mathbb{R}^{\mathbb{Z}}: T_{(q, p)} x=x\right\} .
$$

Two elements of $\mathcal{M}^{\text {per }}(h, \alpha)$ are called $(h)$-neighboring if there does not exist an element of $\mathcal{M}^{\text {per }}(h, \alpha)$ between them. The following two propositions describe the structure of the set $M(h, \alpha)$. For their proofs, see [3].

Proposition 1.7. Suppose that $x^{-}<x^{+}$are $(h)$-neighboring elements of the set $M^{\text {per }}(h, \alpha)$. Then there exist $y^{(1)}, y^{(2)} \in M(h, \alpha)$ such that

$$
\begin{array}{cl}
x^{-}<y^{(1)}<x^{+}, & x^{-}<y^{(2)}<x^{+}, \\
\lim _{i \rightarrow-\infty} y_{i}^{(1)}-x_{i}^{-}=0, & \lim _{i \rightarrow \infty} y_{i}^{(1)}-x_{i}^{+}=0, \\
\lim _{i \rightarrow-\infty} y_{i}^{(2)}-x_{i}^{+}=0, & \lim _{i \rightarrow \infty} y_{i}^{(2)}-x_{i}^{-}=0 .
\end{array}
$$

Suppose that $x^{-}<x^{+}$are $(h)$-neighboring elements of $\mathcal{M}^{\text {per }}(h, \alpha)$. Define

$$
\begin{aligned}
& M^{+}\left(h, \alpha, x^{-}, x^{+}\right)=\left\{x \in M(h, \alpha): \lim _{i \rightarrow-\infty} x_{i}-x_{i}^{-}=0, \lim _{i \rightarrow \infty} x_{i}-x_{i}^{+}=0\right\}, \\
& \mathcal{M}^{-}\left(h, \alpha, x^{-}, x^{+}\right)=\left\{x \in \mathcal{M}(h, \alpha): \lim _{i \rightarrow-\infty} x_{i}-x_{i}^{+}=0, \lim _{i \rightarrow \infty} x_{i}-x_{i}^{-}=0\right\} .
\end{aligned}
$$

We denote by $\mathcal{M}^{+}(h, \alpha)$ (resp., $\left.\mathcal{M}^{-}(h, \alpha)\right)$ the union of the sets $\mathcal{M}^{+}\left(h, \alpha, x^{-}, x^{+}\right)$(resp., $\left.M^{-}\left(h, \alpha, x^{-}, x^{+}\right)\right)$extended over all pairs of $(h)$-neighboring elements $x^{-}<x^{+}$of $M^{\text {per }}(h, \alpha)$. Proposition 1.8. (1) If $x \in \mathcal{M}^{-}\left(h, \alpha, x^{-}, x^{+}\right) \cup M^{+}\left(h, \alpha, x^{-}, x^{+}\right)$, where $x^{-}, x^{+} \in M^{\text {per }}(h, \alpha)$ are $(h)$-neighboring and $x^{-}<x^{+}$, then $x^{-}<x<x^{+}$. 
(2) $\mathcal{M}(h, \alpha)=\mathcal{M}^{\text {per }}(h, \alpha) \cup \mathcal{M}^{+}(h, \alpha) \cup \mathcal{M}^{-}(h, \alpha)$.

(3) The sets $M^{\mathrm{per}}(h, \alpha) \cup \mathcal{M}^{+}(h, \alpha)$ and $M^{\mathrm{per}}(h, \alpha) \cup \mathcal{M}^{-}(h, \alpha)$ are totally ordered.

(4) $\mathcal{M}^{+}(h, \alpha)=\left\{x \in \mathcal{M}(h, \alpha): x>T_{(q, p)} x\right\}$ and $\mathcal{M}^{-}(h, \alpha)=\left\{x \in \mathcal{M}(h, \alpha): x<T_{(q, p)} x\right\}$.

Let $k \geq 2$ be an integer. In this paper, we consider a complete metric space of functions $h: \mathbb{R}^{2} \rightarrow \mathbb{R}^{1}$ which belong to $\mathbb{C}^{k}\left(\mathbb{R}^{2}\right)$. This space is defined in Section 2 and is denoted by $\mathfrak{M}_{k}$. We establish the existence of a set $\mathscr{F} \subset \mathfrak{M}_{k}$ which is a countable intersection of open everywhere dense subsets of $\mathfrak{M}_{k}$ and such that for each $h \in \mathscr{F}$ and each rational $\alpha=p / q$ with $p$ and $q$ relatively prime, the following properties hold:

(i) there exist $(h)$-minimal configurations $x^{(+)}, x^{(-)}$, and $x^{(0)}$ with rotation number $\alpha$ such that $x_{i-q}^{(+)}+p>x_{i}^{(+)}, x_{i-q}^{(-)}+p<x_{i}^{(-)}$, and $x_{i-q}^{(0)}+p=x_{i}^{(0)}$ for all integers $i$;

(ii) any $(h)$-minimal configuration with rotation number $\alpha$ is a translation of one of the configurations $x^{(+)}, x^{(-)}$, and $x^{(0)}$.

\section{Spaces of functions}

Let $k \geq 2$ be an integer. For $f=f\left(x_{1}, x_{2}\right) \in \mathbb{C}^{k}\left(\mathbb{R}^{2}\right)$ and $q=\left(q_{1}, q_{2}\right) \in\{0, \ldots, k\}^{2}$, satisfying $q_{1}+q_{2} \leq k$, we set

$$
|q|=q_{1}+q_{2}, \quad D^{q} f=\frac{\partial^{|q|} f}{\partial x_{1}^{q_{1}} \partial x_{2}^{q_{2}}} .
$$

Denote by $\mathfrak{M}_{k}$ the set of all $h \in \mathbb{C}^{k}\left(\mathbb{R}^{2}\right)$ which have the property (H1), satisfying

$$
\left(\frac{\partial^{2} h}{\partial x_{1} \partial x_{2}}\right)\left(\xi_{1}, \xi_{2}\right) \leq 0 \quad \forall\left(\xi_{1}, \xi_{2}\right) \in \mathbb{R}^{2}
$$

and have the following property:

(H5) there exist $\delta_{h} \in(0,1)$ and $c_{h}>0$ such that

$$
h\left(x_{1}, x_{2}\right) \geq \delta_{h}\left(x_{1}-x_{2}\right)^{2}-c_{h} \quad \forall\left(x_{1}, x_{2}\right) \in \mathbb{R}^{2} .
$$

Clearly (H5) implies (H2).

Denote by $\mathfrak{M}_{k 0}$ the set of all $h \in \mathfrak{M}_{k}$ such that

$$
\left(\frac{\partial^{2} h}{\partial x_{1} \partial x_{2}}\right)\left(\xi_{1}, \xi_{2}\right)<0 \quad \forall\left(\xi_{1}, \xi_{2}\right) \in \mathbb{R}^{2}
$$

For each $N, \epsilon>0$, we set

$$
\begin{aligned}
E_{k}(N, \epsilon)=\{ & \left(h_{1}, h_{2}\right) \in \mathfrak{M}_{k} \times \mathfrak{M}_{k}:\left|D^{q} h_{1}\left(x_{1}, x_{2}\right)-D^{q} h_{2}\left(x_{1}, x_{2}\right)\right| \leq \epsilon \\
& \text { for each } q \in\{0, \ldots, k\}^{2} \text { satisfying }|q| \leq k \\
& \text { and each } \left.\left(x_{1}, x_{2}\right) \in \mathbb{R}^{2} \text { satisfying }\left|x_{1}\right|,\left|x_{2}\right| \leq N\right\} \\
\cap & \left\{\left(h_{1}, h_{2}\right) \in \mathfrak{M}_{k} \times \mathfrak{M}_{k}:\left|h_{1}\left(x_{1}, x_{2}\right)-h_{2}\left(x_{1}, x_{2}\right)\right|<\epsilon\right. \\
& \left.+\epsilon \max \left\{\left|h_{1}\left(x_{1}, x_{2}\right)\right|,\left|h_{2}\left(x_{1}, x_{2}\right)\right|\right\} \forall\left(x_{1}, x_{2}\right) \in \mathbb{R}^{2}\right\} .
\end{aligned}
$$

Using the following simple lemma, we can easily show that for the set $\mathfrak{M}_{k}$ there exists the uniformity which is determined by the base $E_{k}(N, \epsilon), N, \epsilon>0$. 
Lemma 2.1. Let $a, b \in \mathbb{R}^{1}, \epsilon \in(0,1)$, and $|a-b|<\epsilon+\epsilon \max \{|a|,|b|\}$. Then

$$
|a-b|<\epsilon+\epsilon^{2}(1-\epsilon)^{-1}+\epsilon(1-\epsilon)^{-1} \min \{|a|,|b|\} .
$$

It is not difficult to see that the uniformity determined by the base $E_{k}(N, \epsilon), N, \epsilon>0$, is metrizable (by a metric $d_{k}$ ) and complete [9]. For the set $\mathfrak{M}_{k}$, we consider the topology induced by the metric $d_{2}$, which is called the weak topology, and the topology induced by the metric $d_{k}$, which is called the strong topology.

The following result shows that a generic function in $\mathfrak{M}_{k}$ belongs to $\mathfrak{M}_{k 0}$ and by Remark 1.3 has the properties (H1), (H2), (H3), and (H4).

THeOREM 2.2. There exists a set $\mathscr{F}_{0} \subset \mathfrak{M}_{k 0}$ which is a countable intersection of open (in the weak topology) everywhere dense (in the strong topology) subsets of $\mathfrak{M}_{k}$.

Proof. For $h \in \mathfrak{M}_{k}$ and $\gamma \in(0,1)$, define $h_{\gamma}: \mathbb{R}^{2} \rightarrow \mathbb{R}^{1}$ by

$$
h_{\gamma}\left(x_{1}, x_{2}\right)=h\left(x_{1}, x_{2}\right)+\gamma\left(x_{1}-x_{2}\right)^{2}, \quad\left(x_{1}, x_{2}\right) \in \mathbb{R}^{2} \text {. }
$$

It is easy to see that for $h \in \mathfrak{M}_{k}$ and $\gamma \in(0,1), h_{\gamma} \in \mathfrak{M}_{k 0}$ and

$$
\left(\frac{\partial^{2} h_{\gamma}}{\partial x_{1} \partial x_{2}}\right)\left(\xi_{1}, \xi_{2}\right) \leq-2 \gamma, \quad\left(\xi_{1}, \xi_{2}\right) \in \mathbb{R}^{2},
$$

and $h_{\gamma} \rightarrow h$ as $\gamma \rightarrow 0^{+}$in the strong topology.

Let $f \in \mathfrak{M}_{k}$, let $\gamma \in(0,1)$, and let $i \geq 1$ be an integer. By (2.5) and (2.8), there exists an open neighborhood $U(f, \gamma, i)$ of $f_{\gamma}$ in $\mathfrak{M}_{k}$ with the weak topology such that the following property holds:

(P1) for each $g \in \mathcal{U}(f, \gamma, i)$ and each $\left(\xi_{1}, \xi_{2}\right) \in \mathbb{R}^{2}$ satisfying $\left|\xi_{1}\right|,\left|\xi_{2}\right| \leq i$, the inequality $\partial^{2} g / \partial x_{1} \partial x_{2}\left(\xi_{1}, \xi_{2}\right) \leq-\gamma$ holds.

Define $\mathscr{F}_{0}=\cap_{n=1}^{\infty} \cup\left\{\mathcal{U}(f, \gamma, i): f \in \mathfrak{M}_{k}, \gamma \in(0,1), i \geq n\right\}$. Clearly, $\mathscr{F}_{0}$ is a countable intersection of open (in the weak topology) everywhere dense (in the strong topology) subsets of $\mathfrak{M}_{k}$. We will show that $\mathscr{F}_{0} \subset \mathfrak{M}_{k 0}$. Let $h \in \mathscr{F}_{0},\left(\xi_{1}, \xi_{2}\right) \in \mathbb{R}^{2}$. Choose a natural number $n$ such that $\left|\xi_{1}\right|+\left|\xi_{2}\right|<n$. There exist $f \in \mathfrak{M}_{k}, \gamma \in(0,1)$, and an integer $i \geq n$ such that $h \in \mathcal{U}(f, \gamma, i)$. It follows from property (P1) and the choice of $n$ that $\left(\partial^{2} h / \partial x_{1} \partial x_{2}\right)\left(\xi_{1}, \xi_{2}\right) \leq-\gamma$. Therefore, $h \in \mathfrak{M}_{k 0}$. This completes the proof of Theorem 2.2.

\section{The main results}

We will prove the following result.

Theorem 3.1. Let $k \geq 2$ be an integer and $\alpha$ a rational number. Then there exists a set $\mathscr{F}_{\alpha} \subset \mathfrak{M}_{k 0}$ which is a countable intersection of open (in the weak topology) everywhere dense (in the strong topology) subsets of $\mathfrak{M}_{k}$ such that for each $f \in \mathscr{F}_{\alpha}$, the following assertions hold:

(1) if $x, y \in M^{\mathrm{per}}(f, \alpha)$, then there exist integers $m, n$ such that $y_{i}=x_{i-m}+n$ for all $i \in \mathbb{Z}$; 
(2) if $x, y \in M^{+}(f, \alpha)$, then there exist integers $m, n$ such that $y_{i}=x_{i-m}+n$ for all $i \in \mathbb{Z}$;

(3) if $x, y \in M^{-}(f, \alpha)$, then there exist integers $m, n$ such that $y_{i}=x_{i-m}+n$ for all $i \in \mathbb{Z}$.

It is not difficult to see that Theorem 3.1 implies the following result.

Theorem 3.2. Let $k \geq 2$ be an integer. Then there exists a set $\mathscr{F}_{\mathcal{F}} \subset \mathfrak{M}_{k 0}$ which is a countable intersection of open (in the weak topology) everywhere dense (in the strong topology) subsets of $\mathfrak{M}_{k}$ such that for each rational number $\alpha$ and each $f \in \mathscr{F}$, assertions (1), (2), and (3) of Theorem 3.1 hold.

Theorem 3.1 follows from the next two propositions.

Proposition 3.3. Let $k \geq 2$ be an integer and $\alpha$ a rational number. Then there exists a set $\mathscr{F}_{\alpha^{+}} \subset \mathfrak{M}_{k 0}$ which is a countable intersection of open (in the weak topology) everywhere dense (in the strong topology) subsets of $\mathfrak{M}_{k}$ such that for each $f \in \mathscr{F}_{\alpha^{+}}$, assertions (1) and (2) of Theorem 3.1 hold.

Proposition 3.4. Let $k \geq 2$ be an integer and $\alpha$ a rational number. Then there exists a set $\mathscr{F}_{\alpha^{-}} \subset \mathfrak{M}_{k 0}$ which is a countable intersection of open (in the weak topology) everywhere dense (in the strong topology) subsets of $\mathfrak{M}_{k}$ such that for each $f \in \mathscr{F}_{\alpha^{-}}$, assertions (1) and (3) of Theorem 3.1 hold.

Our goal is to prove Proposition 3.3. Proposition 3.4 is proved analogously.

\section{Preliminary results for assertion (1) of Theorem 3.1}

Let $m \geq 1$ be an integer. Consider the manifold $\left(\mathbb{R}^{1} / \mathbb{Z}\right)^{m}$ and the canonical mapping $P_{m}$ : $\mathbb{R}^{m} \rightarrow\left(\mathbb{R}^{1} / \mathbb{Z}\right)^{m}$. We have the following result [21, Proposition 6.2].

Proposition 4.1. Let $\Omega$ be a closed subset of $\left(\mathbb{R}^{1} / \mathbb{Z}\right)^{2}$. Then there exists a nonnegative function $\phi \in \mathbb{C}^{\infty}\left(\left(\mathbb{R}^{1} / \mathbb{Z}\right)^{2}\right)$ such that $\Omega=\left\{x \in\left(\mathbb{R}^{1} / \mathbb{Z}\right)^{2}: \phi(x)=0\right\}$.

Corollary 4.2. Let $\Omega$ be a closed subset of $\mathbb{R}^{1} / \mathbb{Z}$. Then there exists a nonnegative function $\phi \in \mathbb{C}^{\infty}\left(\mathbb{R}^{1} / \mathbb{Z}\right)$ such that $\Omega=\left\{x \in \mathbb{R}^{1} / \mathbb{Z}: \phi(x)=0\right\}$.

In this section, we assume that $k \geq 2$ is an integer and $\alpha=p / q$ is an irreducible fraction, where $q \geq 1$ and $p$ are integers.

For each $f \in \mathfrak{M}_{k 0}$, define

$$
E_{\alpha}(f)=\sum_{i=0}^{q-1} f\left(x_{i}, x_{i+1}\right), \quad x \in \mathcal{M}^{\mathrm{per}}(f, \alpha),
$$

(see Proposition 1.6).

Proposition 4.3. Let $f \in \mathfrak{M}_{k}$, let $Q$ be a natural number, and let $D, \epsilon>0$. Then there exists a neighborhood $U$ of $f$ in $\mathfrak{M}_{k}$ with the weak topology such that for each $g \in \mathcal{U}$, each pair of integers $n_{1}, n_{2} \in\left[n_{1}+1, n_{1}+Q\right]$, and each sequence $\left\{x_{i}\right\}_{i=n_{1}}^{n_{2}} \subset \mathbb{R}^{1}$ which satisfies

$$
\min \left\{\sum_{i=n_{1}}^{n_{2}-1} f\left(x_{i}, x_{i+1}\right), \sum_{i=n_{1}}^{n_{2}-1} g\left(x_{i}, x_{i+1}\right)\right\} \leq D,
$$


the inequality

$$
\left|\sum_{i=n_{1}}^{n_{2}-1} f\left(x_{i}, x_{i+1}\right)-\sum_{i=n_{1}}^{n_{2}-1} g\left(x_{i}, x_{i+1}\right)\right| \leq \epsilon
$$

holds.

Proof. By (H5), there exist $\delta_{0} \in(0,1)$ and $c_{0}>0$ such that

$$
f\left(x_{1}, x_{2}\right) \geq \delta_{0}\left(x_{1}-x_{2}\right)^{2}-c_{0} \quad \forall\left(x_{1}, x_{2}\right) \in \mathbb{R}^{2} .
$$

Choose a positive number $\epsilon_{1}$ for which

$$
\epsilon_{1}\left[Q+c_{0} Q+D\right]<4^{-1} \min \{1, \epsilon\}
$$

and a positive number $\epsilon_{0}<1$ which satisfies

$$
\epsilon_{0}+\epsilon_{0}^{2}\left(1-\epsilon_{0}\right)^{-1}+\epsilon_{0}\left(1-\epsilon_{0}\right)^{-1}<4^{-1} \epsilon_{1}
$$

Define

$$
\mathcal{U}=\left\{g \in \mathfrak{M}_{k}:(f, g) \in E_{k}\left(1, \epsilon_{0}\right)\right\}
$$

(see $(2.5))$.

Assume that $g \in \mathcal{U}, n_{1}, n_{2} \in \mathbb{Z}, n_{2} \in\left[n_{1}+1, n_{1}+Q\right],\left\{x_{i}\right\}_{i=n_{1}}^{n_{2}} \subset \mathbb{R}^{1}$, and that (4.2) holds. By (2.5) and (4.7) for every $\left(z_{1}, z_{2}\right) \in \mathbb{R}^{2}$,

$$
\left|f\left(z_{1}, z_{2}\right)-g\left(z_{1}, z_{2}\right)\right|<\epsilon_{0}+\epsilon_{0} \max \left\{\left|f\left(z_{1}, z_{2}\right)\right|,\left|g\left(z_{1}, z_{2}\right)\right|\right\} .
$$

It follows from (4.6), (4.8), and Lemma 2.1 that for every $\left(z_{1}, z_{2}\right) \in \mathbb{R}^{2}$,

$$
\begin{aligned}
& \left|f\left(z_{1}, z_{2}\right)-g\left(z_{1}, z_{2}\right)\right| \\
& \quad<\epsilon_{0}+\epsilon_{0}^{2}\left(1-\epsilon_{0}\right)^{-1}+\epsilon_{0}\left(1-\epsilon_{0}\right)^{-1} \min \left\{\left|f\left(z_{1}, z_{2}\right)\right|,\left|g\left(z_{1}, z_{2}\right)\right|\right\} \\
& \quad<4^{-1} \epsilon_{1}+4^{-1} \epsilon_{1} \min \left\{\left|f\left(z_{1}, z_{2}\right)\right|,\left|g\left(z_{1}, z_{2}\right)\right|\right\} .
\end{aligned}
$$

Formulas (4.4) and (4.9) imply that for every $\left(z_{1}, z_{2}\right) \in \mathbb{R}^{2}$,

$$
g\left(z_{1}, z_{2}\right) \geq f\left(z_{1}, z_{2}\right)-4^{-1} \epsilon_{1}-4^{-1} \epsilon_{1}\left|f\left(z_{1}, z_{2}\right)\right| \geq-4^{-1} \epsilon_{1}-2 c_{0} .
$$

Set

$$
\lambda_{i}=\min \left\{f\left(x_{i}, x_{i+1}\right), g\left(x_{i}, x_{i+1}\right)\right\}, \quad i=n_{1}, \ldots, n_{2}-1 .
$$

It follows from (4.4), (4.9), (4.10), and (4.11) that for $i=n_{1}, \ldots, n_{2}-1$,

$$
\begin{aligned}
& \left|f\left(x_{i}, x_{i+1}\right)-g\left(x_{i}, x_{i+1}\right)\right| \\
& \quad<4^{-1} \epsilon_{1}+4^{-1} \epsilon_{1} \min \left\{f\left(x_{i}, x_{i+1}\right)+2 c_{0}, g\left(x_{i}, x_{i+1}\right)+4 c_{0}+2\right\} \\
& \quad \leq 4^{-1} \epsilon_{1}+4^{-1} \epsilon_{1} \lambda_{i}+c_{0} \epsilon_{1}+\frac{\epsilon_{1}}{2} .
\end{aligned}
$$


By these inequalities, (4.2), (4.5), and (4.11),

$$
\begin{aligned}
& \left|\sum_{i=n_{1}}^{n_{2}-1} f\left(x_{i}, x_{i+1}\right)-g\left(x_{i}, x_{i+1}\right)\right| \\
& \quad \leq\left(n_{2}-n_{1}\right)\left[4^{-1} \epsilon_{1}+2^{-1} \epsilon_{1}+\epsilon_{1} c_{0}\right]+4^{-1} \epsilon_{1} \sum_{i=n_{1}}^{n_{2}-1} \lambda_{i} \\
& \quad \leq\left(n_{2}-n_{1}\right)\left[\epsilon_{1}+\epsilon_{1} c_{0}\right]+4^{-1} \epsilon_{1} D \\
& \quad \leq Q\left(\epsilon_{1}+\epsilon_{1} c_{0}\right)+4^{-1} \epsilon_{1} D<\epsilon .
\end{aligned}
$$

This completes the proof of Proposition 4.3.

Corollary 4.4. Let $f \in \mathfrak{M}_{k 0}$ and $\epsilon>0$. Then there exists a neighborhood $\mathcal{U}$ of $f$ in $\mathfrak{M}_{k}$ with the weak topology such that for each $g \in \mathcal{U} \cap \mathfrak{M}_{k 0}, E_{\alpha}(g) \leq E_{\alpha}(f)+\epsilon$.

Proposition 4.5. Assume that $f \in \mathfrak{M}_{k 0}, f_{n} \in \mathfrak{M}_{k 0}, n=1,2, \ldots, \lim _{n \rightarrow \infty} f_{n}=f$ in the weak topology,

$$
\begin{gathered}
x^{(n)} \in \mathcal{M}\left(f_{n}\right), \quad n=1,2, \ldots, x \in \mathbb{R}^{\mathbb{Z}}, \\
\lim _{n \rightarrow \infty} x_{i}^{(n)}=x_{i} \quad \forall i \in \mathbb{Z} .
\end{gathered}
$$

Then $x \in \mathcal{M}(f)$.

Proof. We assume the converse. Then, there exist integers $i_{1}<i_{2}$ and a sequence $\left\{y_{i}\right\}_{i=i_{1}}^{i_{2}} \subset$ $\mathbb{R}^{1}$ such that

$$
y_{i_{1}}=x_{i_{1}}, y_{i_{2}}=x_{i_{2}}, \quad \sum_{i=i_{1}}^{i_{2}-1} f\left(y_{i}, y_{i+1}\right)<\sum_{i=i_{1}}^{i_{2}-1} f\left(x_{i}, x_{i+1}\right)
$$

Set

$$
\Delta=\sum_{i=i_{1}}^{i_{2}-1}\left[f\left(x_{i}, x_{i+1}\right)-f\left(y_{i}, y_{i+1}\right)\right]
$$

For each integer $n \geq 1$, define a finite sequence $\left\{y_{i}^{(n)}\right\}_{i=i_{1}}^{i_{2}} \subset \mathbb{R}^{1}$ as follows:

$$
y_{i_{1}}^{(n)}=x_{i_{1}}^{(n)}, \quad y_{i_{2}}^{(n)}=x_{i_{2}}^{(n)}, \quad y_{i}^{(n)}=y_{i}, \quad i \in\left\{i_{1}, \ldots, i_{2}\right\} \backslash\left\{i_{1}, i_{2}\right\} .
$$

It follows from $(4.14),(4.15),(4.16),(4.17)$, and the continuity of $f$ that

$$
\begin{aligned}
\lim _{n \rightarrow \infty}[ & {\left[\sum_{i=i_{1}}^{i_{2}-1} f\left(x_{i}^{(n)}, x_{i+1}^{(n)}\right)-\sum_{i=i_{1}}^{i_{2}-1} f\left(y_{i}^{(n)}, y_{i+1}^{(n)}\right)\right] } \\
& =\sum_{i=i_{1}}^{i_{2}-1} f\left(x_{i}, x_{i+1}\right)-\sum_{i=i_{1}}^{i_{2}-1} f\left(y_{i}, y_{i+1}\right)=\Delta>0 .
\end{aligned}
$$


Formulas (4.14) and (4.18) imply that the sequences

$$
\left\{\sum_{i=i_{1}}^{i_{2}-1} f\left(x_{i}^{(n)}, x_{i+1}^{(n)}\right)\right\}_{n=1}^{\infty}, \quad\left\{\sum_{i=i_{1}}^{i_{2}-1} f\left(y_{i}^{(n)}, y_{i+1}^{(n)}\right)\right\}_{n=1}^{\infty}
$$

are bounded. It follows from this fact, Proposition 4.3, and the equality $f=\lim _{n \rightarrow \infty} f_{n}$ in the weak topology that

$$
\begin{aligned}
& \lim _{n \rightarrow \infty}\left[\sum_{i=i_{1}}^{i_{2}-1} f\left(x_{i}^{(n)}, x_{i+1}^{(n)}\right)-\sum_{i=i_{1}}^{i_{2}-1} f_{n}\left(x_{i}^{(n)}, x_{i+1}^{(n)}\right)\right]=0, \\
& \lim _{n \rightarrow \infty}\left[\sum_{i=i_{1}}^{i_{2}-1} f\left(y_{i}^{(n)}, y_{i+1}^{(n)}\right)-\sum_{i=i_{1}}^{i_{2}-1} f_{n}\left(y_{i}^{(n)}, y_{i+1}^{(n)}\right)\right]=0 .
\end{aligned}
$$

Formulas (4.18) and (4.20) imply that

$$
\lim _{n \rightarrow \infty}\left[\sum_{i=i_{1}}^{i_{2}-1} f_{n}\left(x_{i}^{(n)}, x_{i+1}^{(n)}\right)-\sum_{i=i_{1}}^{i_{2}-1} f_{n}\left(y_{i}^{(n)}, y_{i+1}^{(n)}\right)\right]=\Delta>0 .
$$

There is an integer $n_{0} \geq 1$ such that for each integer $n \geq n_{0}$,

$$
\sum_{i=i_{1}}^{i_{2}-1} f_{n}\left(x_{i}^{(n)}, x_{i+1}^{(n)}\right)-\sum_{i=i_{1}}^{i_{2}-1} f_{n}\left(y_{i}^{(n)}, y_{i+1}^{(n)}\right)>\frac{\Delta}{2} .
$$

This fact contradicts the $\left(f_{n}\right)$-minimality of $x^{(n)}$ for all $n \geq n_{0}$. The contradiction we have reached proves Proposition 4.5.

Proposition 4.6. Let $f \in \mathfrak{M}_{k 0}, f_{n} \in \mathfrak{M}_{k 0}, n=1,2, \ldots, \lim _{n \rightarrow \infty} f_{n}=f$ in the weak topology, $x^{(n)} \in \mathcal{M}^{\mathrm{per}}\left(f_{n}, \alpha\right), n=1,2, \ldots$, and let the sequence $\left\{x_{0}^{(n)}\right\}_{n=1}^{\infty}$ be bounded. Then the following assertions hold:

(1) there exist $x \in \mathbb{R}^{\mathbb{Z}}$ and a strictly increasing sequence of natural numbers $\left\{n_{j}\right\}_{j=1}^{\infty}$ such that

$$
\begin{gathered}
x_{i+q}=x_{i}+p, \quad i \in \mathbb{Z}, \\
x_{i}^{\left(n_{j}\right)} \longrightarrow x_{i} \quad \text { as } j \longrightarrow \infty, \forall i \in \mathbb{Z} ;
\end{gathered}
$$

(2) assume that $x \in \mathbb{R}^{\mathbb{Z}}$ and $\left\{n_{j}\right\}_{j=1}^{\infty}$ is a strictly increasing sequence of natural numbers such that (4.23) and (4.24) hold. Then $x \in \mathcal{M}^{\mathrm{per}}(f, \alpha)$ and

$$
E_{\alpha}(f)=\sum_{i=0}^{q-1} f\left(x_{i}, x_{i+1}\right)=\lim _{j \rightarrow \infty} \sum_{i=0}^{q-1} f_{n_{j}}\left(x_{i}^{\left(n_{j}\right)}, x_{i+1}^{\left(n_{j}\right)}\right)=\lim _{j \rightarrow \infty} E_{\alpha}\left(f_{n_{j}}\right) .
$$

Proof. By Proposition 1.4, the sequence $\left\{x_{i}^{(n)}\right\}_{n=1}^{\infty}$ is bounded for all $i \in \mathbb{Z}$. This fact implies that there exist a strictly increasing sequence of natural numbers $\left\{n_{j}\right\}_{j=1}^{\infty}$ and $x \in \mathbb{R}^{\mathbb{Z}}$ such that (4.23) and (4.24) are valid. Therefore, assertion (1) is true. 
We will prove assertion (2). Assume that $x \in \mathbb{R}^{\mathbb{Z}}$ and $\left\{n_{j}\right\}_{j=1}^{\infty}$ is a strictly increasing sequence of natural numbers such that (4.23) and (4.24) hold. By Proposition 4.5 and (4.23), $x \in \mathcal{M}^{\text {per }}(f, \alpha)$. Since $\lim _{n \rightarrow \infty} f_{n}=f$ in the weak topology, it follows from Corollary 4.4 that the sequence $\left\{E_{\alpha}\left(f_{n}\right)\right\}_{n=1}^{\infty}$ is bounded from above. Therefore, the sequence $\left\{\sum_{i=0}^{q-1} f_{n}\left(x_{i}^{(n)}, x_{i+1}^{(n)}\right)\right\}_{n=1}^{\infty}$ is also bounded from above. It follows from this fact, the equality $\lim _{n \rightarrow \infty} f_{n}=f$ in the weak topology, and Proposition 4.3 that

$$
\lim _{n \rightarrow \infty}\left[\sum_{i=0}^{q-1} f_{n}\left(x_{i}^{(n)}, x_{i+1}^{(n)}\right)-\sum_{i=0}^{q-1} f\left(x_{i}^{(n)}, x_{i+1}^{(n)}\right)\right]=0 .
$$

By (4.1), (4.23), (4.24), (4.26), and Corollary 4.4,

$$
\begin{aligned}
E_{\alpha}(f) & \leq \sum_{i=0}^{q-1} f\left(x_{i}, x_{i+1}\right)=\lim _{j \rightarrow \infty} \sum_{i=0}^{q-1} f\left(x_{i}^{\left(n_{j}\right)}, x_{i+1}^{\left(n_{j}\right)}\right) \\
& =\lim _{j \rightarrow \infty} \sum_{i=0}^{q-1} f_{n_{j}}\left(x_{i}^{\left(n_{j}\right)}, x_{i+1}^{\left(n_{j}\right)}\right)=\lim _{j \rightarrow \infty} E_{\alpha}\left(f_{n_{j}}\right) \leq E_{\alpha}(f) .
\end{aligned}
$$

These relations imply (4.25). Proposition 4.6 is proved.

Proposition 4.6 and Corollary 4.4 imply the following result.

Proposition 4.7. The function $f \rightarrow E_{\alpha}(f)$ is continuous on $\mathfrak{M}_{k 0}$ with the relative weak topology.

Proposition 4.8. Assume that $f \in \mathfrak{M}_{k 0}$ and that the following property holds:

If $x^{(1)}, x^{(2)} \in \mathcal{M}^{\mathrm{per}}(f, \alpha)$, then there exists $n=\left(n_{1}, n_{2}\right) \in \mathbb{Z}^{2}$ such that $x^{(2)}=T_{n} x^{(1)}$.

Then there exists $\bar{n}=\left(\bar{n}_{1}, \bar{n}_{2}\right) \in \mathbb{Z}^{2}$ such that for each $x \in M^{\mathrm{per}}(f, \alpha)$,

$$
T_{\bar{n}} x>x, \quad\left\{y \in \mathcal{M}^{\mathrm{per}}(f, \alpha): x<y<T_{\bar{n}} x\right\}=\varnothing .
$$

Proof. Let $\bar{x} \in M^{\text {per }}(f, \alpha)$. Then

$$
\begin{aligned}
\mathcal{M}^{\text {per }}(f, \alpha) & =\left\{T_{n} \bar{x}: n=\left(n_{1}, n_{2}\right) \in \mathbb{Z}^{2}\right\} \\
& =\left\{T_{n} \bar{x}: n=\left(n_{1}, n_{2}\right) \in \mathbb{Z}^{2}, 0 \leq n_{1} \leq q-1\right\} .
\end{aligned}
$$

Formula (4.29) implies that the set

$$
\left\{y \in \mathcal{M}^{\mathrm{per}}(f, \alpha): \bar{x}<y<T_{(0,1)} \bar{x}\right\}
$$

is either finite or empty. Therefore, there exists $\bar{x}^{+} \in \mathcal{M}^{\operatorname{per}}(f, \alpha)$ such that

$$
\bar{x}<\bar{x}^{+}, \quad\left\{y \in M^{\text {per }}(f, \alpha): \bar{x}<y<\bar{x}^{+}\right\}=\varnothing .
$$

There exists $\bar{n}=\left(\bar{n}_{1}, \bar{n}_{2}\right) \in \mathbb{Z}^{2}$ such that

$$
T_{\bar{n}} \bar{x}=\bar{x}^{+} .
$$


Let $x \in \mathcal{M}^{\text {per }}(f, \alpha)$. There exists $n=\left(n_{1}, n_{2}\right) \in \mathbb{Z}^{2}$ such that

$$
x=T_{n} \bar{x}
$$

Formulas (4.31), (4.32), and (4.33) imply that

$$
\begin{gathered}
T_{\bar{n}} x=T_{\bar{n}}\left(T_{n} \bar{x}\right)=T_{n}\left(T_{\bar{n}} \bar{x}\right)=T_{n} \bar{x}^{+}>T_{n} \bar{x}=x, \\
T_{\bar{n}} x>x .
\end{gathered}
$$

Assume that

$$
y \in M^{\text {per }}(f, \alpha), \quad x<y<T_{\bar{n}} x
$$

Then

$$
T_{-n} x<T_{-n} y<T_{-n}\left(T_{\bar{n}} x\right),
$$

where $-n=\left(-n_{1},-n_{2}\right)$. It follows from (4.36), (4.33), and (4.32) that

$$
\bar{x}<T_{-n} y<T_{\bar{n}}\left(T_{-n} x\right)=T_{\bar{n}} \bar{x}=\bar{x}^{+},
$$

a contradiction (see (4.31)). Therefore,

$$
\left\{y \in \mathcal{M}^{\text {per }}(f, \alpha): x<y<T_{\bar{n}} x\right\}=\varnothing .
$$

This completes the proof of Proposition 4.8.

Corollary 4.9. Assume that $f \in \mathfrak{M}_{k 0}$ and that the following property holds:

If $x^{(1)}, x^{(2)} \in M^{\mathrm{per}}(f, \alpha)$, then there exists $n=\left(n_{1}, n_{2}\right) \in \mathbb{Z}^{2}$ such that $T_{n} x^{(1)}=x^{(2)}$.

Then there exists a number $\kappa>0$ such that for each $x, x^{+} \in M^{\text {per }}(f, \alpha)$ satisfying

$$
x<x^{+}, \quad\left\{y \in M^{\operatorname{per}}(f, \alpha): x<y<x^{+}\right\}=\varnothing,
$$

the inequality $x_{i}^{+}-x_{i}>\kappa$ holds for all $i \in \mathbb{Z}$.

Proposition 4.10. Assume that $f \in \mathfrak{M}_{k 0}, \bar{x} \in \mathcal{M}^{\mathrm{per}}(f, \alpha)$,

$$
\mathcal{M}^{\text {per }}(f, \alpha)=\left\{T_{n} \bar{x}: n=\left(n_{1}, n_{2}\right) \in \mathbb{Z}^{2}\right\},
$$

and $\epsilon>0$. Then there exists a neighborhood $\mathcal{U}$ of $f$ in $\mathfrak{M}_{k}$ with the weak topology such that for each $g \in \mathcal{U} \cap \mathfrak{M}_{k 0}$ and each $x \in \mathcal{M}^{\mathrm{per}}(g, \alpha)$, there is $m=\left(m_{1}, m_{2}\right) \in \mathbb{Z}^{2}$ such that $\left|x_{i}-\left(T_{m} \bar{x}\right)_{i}\right| \leq \epsilon, i \in \mathbb{Z}$.

Proof. We assume the converse. Then there exist a sequence $\left\{f_{j}\right\}_{j=1}^{\infty} \subset \mathfrak{M}_{k 0}$ satisfying $\lim _{j \rightarrow \infty} f_{j}=f$ in the weak topology and a sequence $x^{(j)} \in M^{\text {per }}\left(f_{j}, \alpha\right), j=1,2, \ldots$, such that for each natural number $j$ and each $n=\left(n_{1}, n_{2}\right) \in \mathbb{Z}^{2}$,

$$
\sup \left\{\left|x_{i}^{(j)}-\left(T_{n} \bar{x}\right)_{i}\right|: i \in\{0,1, \ldots, q\}\right\}>\epsilon .
$$


We may assume without loss of generality that the sequence $\left\{x_{0}^{(j)}\right\}_{j=1}^{\infty}$ is bounded. By Proposition 4.6, there exist $x \in \mathcal{M}^{\mathrm{per}}(f, \alpha)$ and a strictly increasing sequence of natural numbers $\left\{j_{s}\right\}_{s=1}^{\infty}$ such that

$$
x_{i}^{\left(j_{s}\right)} \longrightarrow x_{i} \text { as } s \longrightarrow \infty, \forall i \in \mathbb{Z}
$$

By (4.40), there exists $m=\left(m_{1}, m_{2}\right) \in \mathbb{Z}^{2}$ such that $x=T_{m} \bar{x}$. It follows from this equality and (4.42) that $x_{i}^{\left(j_{s}\right)} \rightarrow\left(T_{m} \bar{x}\right)_{i}$ as $s \rightarrow \infty$ for all $i \in \mathbb{Z}$. This fact contradicts (4.41). The contradiction we have reached proves Proposition 4.10 .

\section{Preliminary results for assertion (2) of Theorem 3.1}

In this section, we assume that $k \geq 2$ is an integer and $\alpha=p / q$ is an irreducible fraction, where $q \geq 1$ and $p$ are integers. Assume that $f \in \mathfrak{M}_{k 0}$,

$$
\begin{gathered}
\bar{x}, \bar{x}^{+} \in \mathcal{M}^{\text {per }}(f, \alpha), \quad \bar{x}<\bar{x}^{+}, \\
\left\{y \in \mathcal{M}^{\text {per }}(f, \alpha): \bar{x}<y<\bar{x}^{+}\right\}=\varnothing, \\
\mathcal{M}^{\text {per }}(f, \alpha)=\left\{T_{n} \bar{x}: n=\left(n_{1}, n_{2}\right) \in \mathbb{Z}^{2}\right\} .
\end{gathered}
$$

By Corollary 4.9, there exists a number $\kappa>0$ such that

$$
x_{i}^{+}-x_{i}>2 \kappa, \quad i \in \mathbb{Z},
$$

for each $x, x^{+} \in \mathcal{M}^{\text {per }}(f, \alpha)$ which satisfy (4.39).

Lemma 5.1. Let $\epsilon \in(0, \kappa / 2)$. Then there exists a neighborhood $U$ of $f$ in $\mathfrak{M}_{k 0}$ with the weak topology such that the following property holds:

For each $g \in \mathcal{U} \cap \mathfrak{M}_{k 0}$ and each $y \in \mathcal{M}^{\mathrm{per}}(g, \alpha)$, there exists a unique $x \in \mathcal{M}^{\mathrm{per}}(f, \alpha)$ such that

$$
\left|x_{i}-y_{i}\right|<\epsilon, \quad i \in \mathbb{Z}
$$

Proof. By Proposition 4.10, there exists a neighborhood $\mathcal{U}$ of $f$ in $\mathfrak{M}_{k}$ with the weak topology such that the following property holds: for each $g \in \mathcal{U} \cap \mathfrak{M}_{k 0}$ and each $y \in$ $\mathcal{M}^{\text {per }}(g, \alpha)$, there exists $x \in \mathcal{M}^{\text {per }}(f, \alpha)$ such that (5.5) holds.

Let $g \in \mathcal{U} \cap \mathfrak{M}_{k 0}$,

$$
y \in \mathcal{M}^{\text {per }}(g, \alpha), \quad x^{(1)}, x^{(2)} \in \mathcal{M}^{\text {per }}(f, \alpha), \quad\left|x_{i}^{(j)}-y_{i}\right|<\epsilon, i \in \mathbb{Z}, j=1,2 .
$$

To complete the proof of the lemma, it is sufficient to show that $x^{(1)}=x^{(2)}$. Assume the contrary. We may assume without loss of generality that $x^{(1)}<x^{(2)}$. By our choice of $\kappa$ (see (5.4) and (4.39)) and Proposition 4.8,

$$
\inf \left\{x_{i}^{(2)}-x_{i}^{(1)}: i \in \mathbb{Z}\right\}>2 \kappa
$$


On the other hand, it follows from (5.6) that for all $i \in \mathbb{Z}$,

$$
\left|x_{i}^{(2)}-x_{i}^{(1)}\right| \leq\left|x_{i}^{(2)}-y_{i}\right|+\left|y_{i}-x_{i}^{(1)}\right|<2 \epsilon<\kappa,
$$

a contradiction. The contradiction we have reached proves Lemma 5.1.

Lemma 5.2. Let $\epsilon \in(0, \kappa / 2)$ and let a neighborhood $U$ of $f$ in $\mathfrak{M}_{k}$ with the weak topology be as guaranteed in Lemma 5.1. Assume that

$$
\begin{gathered}
g \in \mathcal{U} \cap \mathfrak{M}_{k 0}, \quad y^{(1)}, y^{(2)} \in \mathcal{M}^{\text {per }}(g, \alpha), \quad y^{(1)}<y^{(2)}, \\
\left\{z \in \mathcal{M}^{\text {per }}(g, \alpha): y^{(1)}<z<y^{(2)}\right\}=\varnothing, \\
x^{(1)}, x^{(2)} \in \mathcal{M}^{\text {per }}(f, \alpha), \quad\left|x_{i}^{(j)}-y_{i}^{(j)}\right|<\epsilon, i \in \mathbb{Z}, j=1,2 .
\end{gathered}
$$

Then either $x^{(1)}=x^{(2)}$ or

$$
x^{(1)}<x^{(2)}, \quad\left\{z \in \mathcal{M}^{\text {per }}(f, \alpha): x^{(1)}<z<x^{(2)}\right\}=\varnothing .
$$

Proof. Assume that $x^{(1)} \neq x^{(2)}$. Formulas (5.9) and (5.11) imply that for all $i \in \mathbb{Z}$,

$$
\begin{gathered}
x_{i}^{(2)}-x_{i}^{(1)}=x_{i}^{(2)}-y_{i}^{(2)}+y_{i}^{(2)}-y_{i}^{(1)}+y_{i}^{(1)}-x_{i}^{(1)}>-2 \epsilon>-\kappa, \\
x_{i}^{(2)}-x_{i}^{(1)}>-\kappa \quad \forall i \in \mathbb{Z} .
\end{gathered}
$$

It follows from this inequality, (5.4), and Proposition 4.8 that $x^{(1)}<x^{(2)}$. To complete the proof of the lemma, we need to show that the set

$$
\left\{z \in \mathcal{M}^{\text {per }}(f, \alpha): x^{(1)}<z<x^{(2)}\right\}=\varnothing .
$$

We assume the converse. Then, by Proposition 4.8 , there exists $x^{(3)} \in \mathcal{M}^{\text {per }}(f, \alpha)$ such that

$$
x^{(1)}<x^{(3)}<x^{(2)}, \quad\left\{z \in M^{\text {per }}(f, \alpha): x^{(1)}<z<x^{(3)}\right\}=\varnothing .
$$

It follows from Proposition 4.8, (5.15), and our choice of $\kappa$ (see (5.4) and (4.39)) that

$$
x_{i}^{(2)}-x_{i}^{(3)}>2 \kappa, \quad x_{i}^{(3)}-x_{i}^{(1)}>2 \kappa, \quad i \in \mathbb{Z} .
$$

Formula (5.3) implies that there exists $m=\left(m_{1}, m_{2}\right) \in \mathbb{Z}^{2}$ for which

$$
x^{(3)}=T_{m} x^{(1)}
$$

Set

$$
y^{(3)}=T_{m} y^{(1)}
$$


Clearly, $y^{(3)} \in \mathcal{M}^{\text {per }}(g, \alpha)$. It follows from (5.11), (5.16), (5.17), and (5.18) that for all $i \in \mathbb{Z}$,

$$
\begin{aligned}
y_{i}^{(3)}-y_{i}^{(1)} & =y_{i}^{(3)}-x_{i}^{(3)}+x_{i}^{(3)}-x_{i}^{(1)}+x_{i}^{(1)}-y_{i}^{(1)} \\
& =y_{i-m_{1}}^{(1)}+m_{2}-\left(x_{i-m_{1}}^{(1)}+m_{2}\right)+x_{i}^{(3)}-x_{i}^{(1)}+x_{i}^{(1)}-y_{i}^{(1)} \\
& >-2 \epsilon+2 \kappa>\kappa .
\end{aligned}
$$

Analogously, it follows from (5.11), (5.16), (5.17), and (5.18) that for all $i \in \mathbb{Z}$,

$$
\begin{aligned}
y_{i}^{(2)}-y_{i}^{(3)} & =y_{i}^{(2)}-x_{i}^{(2)}+x_{i}^{(2)}-x_{i}^{(3)}+x_{i}^{(3)}-y_{i}^{(3)} \\
& =y_{i}^{(2)}-x_{i}^{(2)}+x_{i}^{(2)}-x_{i}^{(3)}+x_{i-m_{1}}^{(1)}+m_{2}-\left(y_{i-m_{1}}^{(1)}+m_{2}\right) \\
& >-2 \epsilon+2 \kappa>\kappa .
\end{aligned}
$$

Therefore, $y^{(1)}<y^{(3)}<y^{(2)}$. This fact contradicts (5.10). The contradiction we have reached proves Lemma 5.2.

Definition 5.3. Let $\epsilon \in(0, \kappa / 2), g \in \mathfrak{M}_{k 0}, y \in \mathcal{M}^{+}(g, \alpha), y^{+}, y^{-} \in \mathcal{M}^{\mathrm{per}}(g, \alpha)$,

$$
y^{-}<y<y^{+}, \quad \lim _{i \rightarrow \infty} y_{i}-y_{i}^{+}=0, \quad \lim _{i \rightarrow-\infty} y_{i}-y_{i}^{-}=0 \text {. }
$$

We say that $y$ is regular with respect to $(\epsilon, g)$ if there exist $x^{-}, x^{+} \in M^{\text {per }}(f, \alpha)$ such that

$$
\begin{gathered}
\left|x_{i}^{-}-y_{i}^{-}\right|<\epsilon, \quad\left|x_{i}^{+}-y_{i}^{+}\right|<\epsilon, \quad i \in \mathbb{Z}, \\
x^{-}<x^{+}, \quad\left\{z \in \mathcal{M}^{\operatorname{per}}(f, \alpha): x^{-}<z<x^{+}\right\}=\varnothing .
\end{gathered}
$$

We assume that there exists $\hat{x} \in \mathcal{M}^{+}(f, \alpha)$ such that

$$
\begin{gathered}
\bar{x}<\hat{x}<\bar{x}^{+}, \\
\mathcal{M}^{+}(f, \alpha)=\left\{T_{n} \hat{x}: n=\left(n_{1}, n_{2}\right) \in \mathbb{Z}^{2}\right\} .
\end{gathered}
$$

Lemma 5.4. Let a neighborhood $U$ of $f$ in $\mathfrak{M}_{k}$ with the weak topology be as guaranteed in Lemma 5.1 with $\epsilon=\kappa / 4$. Assume that $\left\{f_{n}\right\}_{n=1}^{\infty} \subset \mathcal{U} \cap \mathfrak{M}_{k 0}, \lim _{n \rightarrow \infty} f_{n}=f$ in the weak topology, and that $x^{(n)} \in \mathcal{M}^{+}\left(f_{n}, \alpha\right)$ is regular with respect to $\left(\kappa / 4, f_{n}\right), n=1,2, \ldots$ Then there exist a strictly increasing sequence of natural numbers $\left\{n_{j}\right\}_{j=1}^{\infty}$ and a sequence $s^{(j)}=$ $\left(s_{1}^{(j)}, s_{2}^{(j)}\right) \in \mathbb{Z}^{2}, j=1,2, \ldots$, such that

$$
T_{s^{(j)}} x_{i}^{\left(n_{j}\right)} \longrightarrow \hat{x}_{i} \quad \text { as } j \longrightarrow \infty, \forall i \in \mathbb{Z} .
$$

Proof. By (5.1), (5.2), and (5.23),

$$
\lim _{i \rightarrow-\infty} \hat{x}_{i}-\bar{x}_{i}=0, \quad \lim _{i \rightarrow \infty} \hat{x}_{i}-\bar{x}_{i}^{+}=0 .
$$

Let $n \geq 1$ be an integer. There exist

$$
x^{\left(n^{+}\right)}, x^{\left(n^{-}\right)} \in M^{\text {per }}\left(f_{n}, \alpha\right)
$$


such that

$$
\begin{gathered}
x^{\left(n^{-}\right)}<x^{(n)}<x^{\left(n^{+}\right)}, \\
\lim _{i \rightarrow-\infty} x_{i}^{\left(n^{-}\right)}-x_{i}^{(n)}=0, \quad \lim _{i \rightarrow \infty} x_{i}^{\left(n^{+}\right)}-x_{i}^{(n)}=0 .
\end{gathered}
$$

Since $f_{n} \in \mathcal{U}$, it follows from the definition of $U$ and Lemma 5.1 that there exist unique $z^{\left(n^{-}\right)}, z^{\left(n^{+}\right)} \in M^{\text {per }}(f, \alpha)$ such that

$$
\left|z_{i}^{\left(n^{-}\right)}-x_{i}^{\left(n^{-}\right)}\right| \leq \frac{\kappa}{4}, \quad\left|z_{i}^{\left(n^{+}\right)}-x_{i}^{\left(n^{+}\right)}\right|<\frac{\kappa}{4}, \quad i \in \mathbb{Z}
$$

Since $x^{(n)}$ is regular with respect to $\left(\kappa / 4, f_{n}\right)$, we have

$$
z^{\left(n^{-}\right)}<z^{\left(n^{+}\right)}, \quad\left\{z \in M^{\text {per }}(f, \alpha): z^{\left(n^{-}\right)}<z<z^{\left(n^{+}\right)}\right\}=\varnothing .
$$

Since $\lim _{n \rightarrow \infty} f_{n}=f$ in the weak topology, it follows from Lemma 5.1 that

$$
\lim _{n \rightarrow \infty} \sup \left\{\left|z_{i}^{\left(n^{-}\right)}-x_{i}^{\left(n^{-}\right)}\right|,\left|z_{i}^{\left(n^{+}\right)}-x_{i}^{\left(n^{+}\right)}\right|: i \in \mathbb{Z}\right\}=0
$$

It follows from (5.1), (5.2), (5.3), (5.31), and Proposition 4.8 that there is $l \in \mathbb{Z}^{2}$ such that $z^{\left(n^{-}\right)}=T_{l} \bar{x}$ and $z^{\left(n^{+}\right)}=T_{l} \bar{x}^{+}$. We may assume without loss of generality that

$$
z^{\left(n^{-}\right)}=\bar{x}, \quad z^{\left(n^{+}\right)}=\bar{x}^{+}, \quad n=1,2, \ldots
$$

It follows from (5.30), (5.33), and the definition of $\kappa$ (see (5.4) and (4.39)) that for any integer $n \geq 1$ and any integer $i$,

$$
\begin{gathered}
x_{i}^{\left(n^{+}\right)}-x_{i}^{\left(n^{-}\right)} \geq x_{i}^{\left(n^{+}\right)}-z_{i}^{\left(n^{+}\right)}+z_{i}^{\left(n^{+}\right)}-z_{i}^{\left(n^{-}\right)}+z_{i}^{\left(n^{-}\right)}-x_{i}^{\left(n^{-}\right)}>-\frac{\kappa}{2}+\bar{x}_{i}^{+}-\bar{x}_{i}>\frac{3 \kappa}{2}, \\
x_{i}^{\left(n^{+}\right)}-x_{i}^{\left(n^{-}\right)}>\frac{3 \kappa}{2} .
\end{gathered}
$$

Let $n \geq 1$ be an integer. It follows from (5.28), (5.29), and (5.35) that there exists an integer $t_{n}$ such that

$$
x_{t_{n}}^{(n)}-x_{t_{n}}^{\left(n^{-}\right)} \leq \frac{\kappa}{2}, \quad x_{t_{n}+1}^{(n)}-x_{t_{n}+1}^{\left(n^{-}\right)}>\frac{\kappa}{2} .
$$

By using translations, we may assume without loss of generality that

$$
t_{n} \in[0, q]
$$

Formulas (5.28), (5.30), and (5.33) imply that for all integers $n \geq 1$ and all $i \in \mathbb{Z}$,

$$
\bar{x}_{i}-\frac{\kappa}{4}<x_{i}^{\left(n^{-}\right)}<x_{i}^{(n)}<x_{i}^{\left(n^{+}\right)}<\bar{x}_{i}^{+}+\frac{\kappa}{4} .
$$


Therefore, for any $i \in \mathbb{Z}$, the sequence $\left\{x_{i}^{(n)}\right\}_{n=1}^{\infty}$ is bounded. Together with (5.37), this implies that there exist $u \in \mathbb{R}^{\mathbb{Z}}$ and a strictly increasing sequence of natural numbers $\left\{n_{j}\right\}_{j=1}^{\infty}$ such that

$$
x_{i}^{\left(n_{j}\right)} \longrightarrow u_{i} \quad \text { as } j \longrightarrow \infty, \forall i \in \mathbb{Z}, \quad t_{n_{j}}=t_{n_{1}}, \quad j=1,2, \ldots
$$

It follows from (5.28), (5.32), (5.33), and (5.39) that for all $i \in \mathbb{Z}$,

$$
u_{i}=\lim _{j \rightarrow \infty} x_{i}^{\left(n_{j}\right)} \in\left[\lim _{j \rightarrow \infty} x_{i}^{\left(n_{j}^{-}\right)}, \lim _{j \rightarrow \infty} x_{i}^{\left(n_{j}^{+}\right)}\right]=\left[\bar{x}_{i}, \bar{x}_{i}^{+}\right] .
$$

By Proposition 4.5, $u \in M(f)$. Since $x^{(n)} \in \mathcal{M}^{+}\left(f_{n}, \alpha\right), n=1,2, \ldots$, we have $x^{(n)}>T_{(q, p)} x^{(n)}$, $n=1,2, \ldots$ Therefore, $x_{i}^{(n)}>x_{i-q}^{(n)}+p$ for any integer $n \geq 1$ and any integer $i$. Combined with (5.39), this fact implies that $u_{i} \geq u_{i-q}+p$ for all $i \in \mathbb{Z}$ and that

$$
u \in M^{\text {per }}(f, \alpha) \cup M^{+}(f, \alpha) .
$$

It follows from (5.36), (5.39), and (5.40) that

$$
\begin{array}{r}
u_{t_{1}}-\bar{x}_{t_{1}}=\lim _{j \rightarrow \infty} x_{t_{1}}^{\left(n_{j}\right)}-\lim _{j \rightarrow \infty} x_{t_{1}}^{\left(n_{j}^{-}\right)} \leq \frac{\kappa}{2}, \\
u_{t_{1}+1}-\bar{x}_{t_{1}+1}=\lim _{j \rightarrow \infty} x_{t_{1}+1}^{\left(n_{j}\right)}-\lim _{j \rightarrow \infty} x_{t_{1}+1}^{\left(n_{j}^{-}\right)} \geq \frac{\kappa}{2} .
\end{array}
$$

By these relations, (5.40), the definition of $\kappa$ (see (5.4) and (4.39)), (5.1), and (5.2), $u \notin\left\{\bar{x}, \bar{x}^{+}\right\}$. Combined with (5.1), (5.2), (5.40), and (5.41), this fact implies that $u \in$ $\mathcal{M}^{+}(f, \alpha)$. By (5.24), there exists $m=\left(m_{1}, m_{2}\right) \in \mathbb{Z}^{2}$ such that $T_{m} x=\hat{x}$. This completes the proof of Lemma 5.4 .

Lemma 5.5. Let $Q \geq 1$ be an integer and $\epsilon \in(0, \kappa / 4)$. Then there exists a neighborhood $U$ of $f$ in $\mathfrak{M}_{k}$ with the weak topology such that for each $g \in \mathcal{U} \cap \mathfrak{M}_{k 0}$ and each $y \in \mathcal{M}^{+}(g, \alpha)$, one of the following properties holds:

(a) there exists $n=\left(n_{1}, n_{2}\right) \in \mathbb{Z}^{2}$ such that

$$
\left|\left(T_{n} y\right)_{i}-\bar{x}_{i}\right|<\epsilon, \quad i \in \mathbb{Z}
$$

(b) there exists $n=\left(n_{1}, n_{2}\right) \in \mathbb{Z}^{2}$ such that

$$
\left|\left(T_{n} y\right)_{i}-\hat{x}_{i}\right|<\epsilon, \quad i=-Q, \ldots, Q .
$$

Proof. Assume the contrary. Then there exist a sequence $\left\{f_{s}\right\}_{s=1} \subset \mathfrak{M}_{k 0}$, such that $\lim _{s \rightarrow \infty} f_{s}=f$ in the weak topology, and a sequence $y^{(s)} \in \mathcal{M}^{+}\left(f_{s}, \alpha\right), s=1,2, \ldots$, such that for any integer $s \geq 1$, the following properties hold:

(c) for any $n=\left(n_{1}, n_{2}\right) \in \mathbb{Z}^{2}$,

$$
\sup \left\{\left|\left(T_{n} y^{(s)}\right)_{i}-\bar{x}_{i}\right|: i \in \mathbb{Z}\right\} \geq \epsilon ;
$$


(d) for any $n=\left(n_{1}, n_{2}\right) \in \mathbb{Z}^{2}$,

$$
\sup \left\{\left|\left(T_{n} y^{(s)}\right)_{i}-\hat{x}_{i}\right|: i=-Q, \ldots, Q\right\} \geq \epsilon .
$$

By Lemmas 5.1 and 5.2 and (5.3), $y^{(s)}$ is regular with respect to $\left(f_{s}, \epsilon / 2\right)$ for all sufficiently large integers $s$.

By Lemma 5.4, there exist a strictly increasing sequence of natural numbers $\left\{s_{j}\right\}_{j=1}^{\infty}$ and a sequence $n^{(j)}=\left(n_{1}^{(j)}, n_{2}^{(j)}\right) \in \mathbb{Z}^{2}, j=1,2, \ldots$, such that $\left(T_{n^{(j)}} y^{\left(s_{j}\right)}\right)_{i} \rightarrow \hat{x}_{i}$ as $j \rightarrow \infty$ for all $i \in \mathbb{Z}$, a contradiction (see (d)). The contradiction we have reached proves Lemma 5.5.

Lemma 5.6. Let $\epsilon \in(0, \kappa / 4)$. Then there exists a neighborhood $U$ of $f$ in $\mathfrak{M}_{k}$ with the weak topology such that for each $g \in \mathcal{U} \cap \mathfrak{M}_{k 0}$ and each $y \in \mathcal{M}^{+}(g, \alpha)$, one of the following properties holds:

(i) there exists $m=\left(m_{1}, m_{2}\right) \in \mathbb{Z}^{2}$ such that

$$
\left|\left(T_{m} y\right)_{i}-\bar{x}_{i}\right|<\epsilon, \quad i \in \mathbb{Z}
$$

(ii) there exists $m=\left(m_{1}, m_{2}\right) \in \mathbb{Z}^{2}$ such that

$$
\left|\left(T_{m} y\right)_{i}-\hat{x}_{i}\right|<\epsilon, \quad i \in \mathbb{Z}
$$

Proof. Choose a positive number

$$
\epsilon_{0}<\min \left\{\frac{\epsilon}{6}, \frac{\kappa}{8}\right\}
$$

By (5.1), (5.2), and (5.23), there exists a natural number $Q>8 q+8$ such that

$$
\begin{aligned}
& \left|\hat{x}_{i}-\bar{x}_{i}^{+}\right|<\frac{\epsilon_{0}}{4} \quad \forall i \geq \frac{Q}{2}, \\
& \left|\hat{x}_{i}-\bar{x}_{i}\right|<\frac{\epsilon_{0}}{4} \quad \forall i \leq-\frac{Q}{2} .
\end{aligned}
$$

By Lemmas 5.1 and 5.2, there exists a neighborhood $\bigcup_{1}$ of $f$ in $\mathfrak{M}_{k}$ with the weak topology such that the following properties hold:

(iii) for each $g \in \mathfrak{U}_{1} \cap \mathfrak{M}_{k 0}$ and each $y \in \mathcal{M}^{\mathrm{per}}(g, \alpha)$, there exists a unique $x \in$ $\mathcal{M}^{\text {per }}(f, \alpha)$ such that $\left|x_{i}-y_{i}\right|<\epsilon_{0}$ for all $i \in \mathbb{Z}$;

(iv) let $g \in \mathcal{U}_{1} \cap \mathfrak{M}_{k 0}, y^{(1)}, y^{(2)} \in \mathcal{M}^{\text {per }}(g, \alpha)$,

$$
\begin{gathered}
y^{(1)}<y^{(2)}, \quad\left\{z \in \mathcal{M}^{\text {per }}(g, \alpha): y^{(1)}<z<y^{(2)}\right\}=\varnothing, \\
x^{(1)}, x^{(2)} \in \mathcal{M}^{\text {per }}(f, \alpha), \quad\left|x_{i}^{(j)}-y_{i}^{(j)}\right|<\epsilon_{0}, i \in \mathbb{Z}, j=1,2 .
\end{gathered}
$$

Then either $x^{(1)}=x^{(2)}$ or

$$
x^{(1)}<x^{(2)}, \quad\left\{z \in \mathcal{M}^{\text {per }}(f, \alpha): x^{(1)}<z<x^{(2)}\right\}=\varnothing .
$$


By Lemma 5.5, there exists a neighborhood $\mathcal{U}$ of $f$ in $\mathfrak{M}_{k}$ with the weak topology such that $U \subset \mathcal{U}_{1}$ and that for each $g \in \mathcal{U} \cap \mathfrak{M}_{k 0}$ and each $y \in \mathcal{M}^{+}(g, \alpha)$, one of the following properties holds:

(v) there exists $m=\left(m_{1}, m_{2}\right) \in \mathbb{Z}^{2}$ such that $\left|\left(T_{m} y\right)_{i}-\bar{x}_{i}\right|<\epsilon_{0}$ for all $i \in \mathbb{Z}$;

(vi) there exists $m=\left(m_{1}, m_{2}\right) \in \mathbb{Z}^{2}$ such that $\left|\left(T_{m} y\right)_{i}-\hat{x}_{i}\right|<\epsilon_{0}, i=-Q, \ldots, Q$.

Let

$$
g \in \mathcal{U} \cap \mathfrak{M}_{k 0}, \quad y \in \mathcal{M}^{+}(g, \alpha)
$$

If (v) is true, then (ii) also holds. Therefore, we may assume that (v) does not hold. Then, by the definition of $U$ and (5.54), property (vi) holds. We may assume without loss of generality that (vi) holds with $m=(0,0)$. Thus

$$
\left|y_{i}-\hat{x}_{i}\right|<\epsilon_{0}, \quad i=-Q, \ldots, Q
$$

There exist

$$
y^{-}, y^{+} \in \mathcal{M}^{\text {per }}(g, \alpha)
$$

such that

$$
y^{-}<y<y^{+}, \quad \lim _{i \rightarrow-\infty} y_{i}^{-}-y_{i}=0, \quad \lim _{i \rightarrow \infty} y_{i}^{+}-y_{i}=0 .
$$

By property (iii), (5.54), and (5.56), there exist unique

$$
x^{-}, x^{+} \in M^{\text {per }}(f, \alpha)
$$

such that

$$
\left|x_{i}^{-}-y_{i}^{-}\right|<\epsilon_{0}, \quad\left|x_{i}^{+}-y_{i}^{+}\right|<\epsilon_{0}, \quad i \in \mathbb{Z} .
$$

By property (iv), (5.54), (5.56), (5.57), (5.58), and (5.59), either $x^{-}=x^{+}$or

$$
x^{-}<x^{+}, \quad\left\{z \in \mathcal{M}^{\text {per }}(f, \alpha): x^{-}<z<x^{+}\right\}=\varnothing .
$$

If $x^{-}=x^{+}$, then (5.57) and (5.59) imply that for all $i \in \mathbb{Z}$,

$$
\begin{gathered}
y_{i}-x_{i}^{+}=y_{i}-y_{i}^{+}+y_{i}^{+}-x_{i}^{+}<y_{i}^{+}-x_{i}^{+}<\epsilon_{0}, \\
y_{i}-x_{i}^{+}=y_{i}-x_{i}^{-}=y_{i}-y_{i}^{-}+y_{i}^{-}-x_{i}^{-}>y_{i}^{-}-x_{i}^{-}>-\epsilon_{0}, \\
\\
\left|y_{i}-x_{i}^{+}\right|<\epsilon_{0},
\end{gathered}
$$

and combining with (5.3) implies that property (v) holds. The contradiction we have reached proves that (5.60) holds. It follows from (5.57) and (5.59) that for all $i \in \mathbb{Z}$,

$$
x_{i}^{-}-\epsilon_{0}<y_{i}^{-}<y_{i}<y_{i}^{+}<x_{i}^{+}+\epsilon_{0} .
$$

We show that $x^{+}=\bar{x}^{+}$and $x^{-}=\bar{x}$. 
By (5.50) and (5.55) for $i=Q-4 q, \ldots, Q$,

$$
\left|y_{i}-\bar{x}_{i}^{+}\right| \leq\left|y_{i}-\hat{x}_{i}\right|+\left|\hat{x}_{i}-\bar{x}_{i}^{+}\right|<\epsilon_{0}+\frac{\epsilon_{0}}{4},
$$

and for $i=-Q, \ldots,-Q+4 q$,

$$
\left|y_{i}-\bar{x}_{i}\right| \leq\left|y_{i}-\hat{x}_{i}\right|+\left|\hat{x}_{i}-\bar{x}_{i}\right|<\epsilon_{0}+\frac{\epsilon_{0}}{4} .
$$

It follows from (5.62), (5.63), and (5.64) that for $i=Q-4 q, \ldots, Q$,

$$
\bar{x}_{i}^{+}-\epsilon_{0}-\frac{\epsilon_{0}}{4}<y_{i}<x_{i}^{+}+\epsilon_{0}
$$

and that for $i=-Q, \ldots,-Q+4 q$,

$$
x_{i}^{-}-\epsilon_{0}<y_{i}<\bar{x}_{i}+\epsilon_{0}+\frac{\epsilon_{0}}{4} .
$$

Thus

$$
\begin{array}{ll}
\bar{x}_{i}^{+}<x_{i}^{+}+2 \epsilon_{0}+\frac{\epsilon_{0}}{4}, & i=Q-4 q, \ldots, Q, \\
x_{i}^{-}<\bar{x}_{i}+2 \epsilon_{0}+\frac{\epsilon_{0}}{4}, & i=-Q, \ldots,-Q+4 q .
\end{array}
$$

It follows from these inequalities, the relation $Q>8 q+8,(5.1),(5.49),(5.58)$, and the definition of $\kappa$ (see (5.4) and (4.39)) that

$$
\begin{array}{ccc}
\bar{x}^{+}<x^{+} & \text {or } & \bar{x}^{+}=x^{+}, \\
\bar{x}^{-}<\bar{x} & \text { or } & x^{-}=\bar{x} .
\end{array}
$$

Combined with (5.1), (5.2), (5.3), (5.58), and (5.60), this fact implies that

$$
\begin{gathered}
\bar{x}=x^{-}, \quad \bar{x}^{+}=x^{+} \quad \text { or } \quad \bar{x}^{+}<x^{+}, \quad \bar{x}<x^{-}, \\
\text {or } \quad x^{+}<\bar{x}^{+}, \quad x^{-}<\bar{x} .
\end{gathered}
$$

By (5.68) and (5.69),

$$
\bar{x}=x^{-}, \quad x^{+}=\bar{x}^{+} .
$$

We will show that

$$
\left|y_{i}-\hat{x}_{i}\right|<\epsilon
$$

for all $i \in \mathbb{Z}$. By (5.55), it is sufficient to show that (5.71) is valid for all integers $i$ satisfying $|i|>Q$.

Assume that an integer $i>Q$. Then there exist integers $s$ and $j$ such that

$$
s>1, \quad j \in[Q-2 q, Q-q], \quad i=j+s q .
$$


By (5.55),

$$
\left|y_{j}-\hat{x}_{j}\right|<\epsilon_{0}
$$

It follows from (5.50) that

$$
\left|\hat{x}_{i}-\bar{x}_{i}^{+}\right|<\frac{\epsilon_{0}}{4}, \quad\left|\hat{x}_{j}-\bar{x}_{j}^{+}\right|<\frac{\epsilon_{0}}{4} .
$$

By (5.54), (5.56), (5.57), (5.59), (5.70), (5.73), and (5.74),

$$
\begin{gathered}
0<y_{j}^{+}-y_{j}=y_{j}^{+}-x_{j}^{+}+x_{j}^{+}-\hat{x}_{j}+\hat{x}_{j}-y_{j}<\epsilon_{0} \\
+\bar{x}_{j}^{+}-\hat{x}_{j}+\hat{x}_{j}-y_{j}<\epsilon_{0}+\frac{\epsilon_{0}}{4}+\epsilon_{0}<3 \epsilon_{0}, \\
0<y_{j}^{+}-y_{j}<3 \epsilon_{0} .
\end{gathered}
$$

Since $y \in \mathcal{M}^{+}(g, \alpha)$, it follows from (5.56), (5.57), (5.72), and (5.76) that

$$
\begin{aligned}
3 \epsilon_{0} & >y_{j}^{+}-y_{j}>y_{j}^{+}-\left(T_{(-q,-p)} y\right)_{j}>y_{j}^{+}-\left(\left(T_{(-q,-p)}\right)^{s} y\right)_{j} \\
& =y_{j}^{+}-y_{j+s q}+s p=y_{j+s q}^{+}-y_{j+s q}=y_{i}^{+}-y_{i}>0 .
\end{aligned}
$$

Thus, we have shown that

$$
0<y_{i}^{+}-y_{i}<3 \epsilon_{0} \quad \forall i>Q
$$

By (5.59), (5.74), and (5.78) for all integers $i>Q$,

$$
\left|\hat{x}_{i}-y_{i}\right| \leq\left|\hat{x}_{i}-\bar{x}_{i}^{+}\right|+\left|\bar{x}_{i}^{+}-y_{i}^{+}\right|+\left|y_{i}^{+}-y_{i}\right|<\frac{\epsilon_{0}}{4}+\epsilon_{0}+3 \epsilon_{0}
$$

and $\left|\hat{x}_{i}-y_{i}\right|<5 \epsilon_{0}<\epsilon$.

Analogously, we show that (5.71) holds for all integers $i<-Q$. Assume that $i<-Q$ is an integer. Then there exist integers $s$ and $j$ such that

$$
s>1, \quad j \in[-Q+q,-Q+2 q], \quad i=j-s q .
$$

By (5.55), inequality (5.73) is valid. It follows from (5.51) that

$$
\left|\hat{x}_{i}-\bar{x}_{i}\right|<\frac{\epsilon_{0}}{4}, \quad\left|\hat{x}_{j}-\bar{x}_{j}\right|<\frac{\epsilon_{0}}{4} .
$$

By (5.57), (5.59), (5.70), (5.73), and (5.81),

$$
\begin{gathered}
0<y_{j}-y_{j}^{-}=y_{j}-\hat{x}_{j}+\hat{x}_{j}-\bar{x}_{j}+\bar{x}_{j}-y_{j}^{-}<\epsilon_{0}+\frac{\epsilon_{0}}{4}+\bar{x}_{j}-y_{j}^{-} \\
=\epsilon_{0}+\frac{\epsilon_{0}}{4}+x_{j}^{-}-y_{j}^{-}<\epsilon_{0}+\frac{\epsilon_{0}}{4}+\epsilon_{0}<3 \epsilon_{0}, \\
0<y_{j}-y_{j}^{-}<3 \epsilon_{0} .
\end{gathered}
$$


Since $y \in \mathcal{M}^{+}(g, \alpha)$, it follows from (5.56), (5.57), (5.80), and (5.83) that

$$
\begin{aligned}
3 \epsilon_{0} & >y_{j}-y_{j}^{-}>\left(T_{(q, p)} y\right)_{j}-y_{j}^{-}>\left(\left(T_{(q, p)}\right)^{s} y\right)_{j}-y_{j}^{-} \\
& =y_{j-s q}+s p-y_{j}^{-}=y_{j-s q}-y_{j-s q}^{-}=y_{i}-y_{i}^{-}>0 .
\end{aligned}
$$

Thus, we have shown that

$$
0<y_{i}-y_{i}^{-}<3 \epsilon_{0}
$$

It follows from this inequality, (5.59), (5.70), and (5.81) that for all integers $i<-Q$,

$$
\begin{aligned}
\left|\hat{x}_{i}-y_{i}\right| & \leq\left|\hat{x}_{i}-\bar{x}_{i}\right|+\left|\bar{x}_{i}-y_{i}^{-}\right|+\left|y_{i}^{-}-y_{i}\right| \\
& <\frac{\epsilon_{0}}{4}+\epsilon_{0}+3 \epsilon_{0}<5 \epsilon_{0}<\epsilon .
\end{aligned}
$$

This completes the proof of Lemma 5.6.

\section{Proof of Proposition 3.3}

Let $k \geq 2$ be an integer and $\alpha=p / q$ an irreducible fraction, where $q \geq 1$ and $p$ are integers.

Let $f \in \mathfrak{M}_{k 0}$. Choose $x^{(f)} \in \mathcal{M}^{(\text {per })}(f, \alpha)$ such that $\left|x_{0}^{(f)}\right| \leq 1$. By Corollary 4.2, there exists a nonnegative function $\phi_{f} \in \mathbb{C}^{\infty}\left(\left(\mathbb{R}^{1} / \mathbb{Z}\right)\right)$ such that

$$
\left\{z \in \mathbb{R}^{1} / \mathbb{Z}: \phi_{f}(z)=0\right\}=\left\{P_{1}\left(x_{i}^{(f)}\right): i \in \mathbb{Z}\right\}
$$

Let $\gamma \in(0,1)$. Define $f_{\gamma}: \mathbb{R}^{2} \rightarrow \mathbb{R}^{1}$ by

$$
f_{\gamma}\left(\xi_{1}, \xi_{2}\right)=f\left(\xi_{1}, \xi_{2}\right)+\gamma \phi_{f}\left(P_{1}\left(\xi_{1}\right)\right), \quad\left(\xi_{1}, \xi_{2}\right) \in \mathbb{R}^{2}
$$

It is not difficult to see that $f_{\gamma} \in \mathfrak{M}_{k 0}$. It follows from (4.1), (6.1), and (6.2) that

$$
\begin{aligned}
E_{\alpha}(f) & \leq E_{\alpha}\left(f_{\gamma}\right) \leq \sum_{i=0}^{q-1} f_{\gamma}\left(x_{i}^{(f)}, x_{i+1}^{(f)}\right) \\
& =\sum_{i=0}^{q-1} f\left(x_{i}^{(f)}, x_{i+1}^{(f)}\right)+\gamma \sum_{i=0}^{q-1} \phi_{f}\left(P_{1}\left(x_{i}^{(f)}\right)\right) \\
& =\sum_{i=0}^{q-1} f\left(x_{i}^{(f)}, x_{i+1}^{(f)}\right)=E_{\alpha}(f)
\end{aligned}
$$

and that

$$
E_{\alpha}(f)=E_{\alpha}\left(f_{\gamma}\right)=\sum_{i=0}^{q-1} f_{\gamma}\left(x_{i}^{(f)}, x_{i+1}^{(f)}\right)=\sum_{i=0}^{q-1} f\left(x_{i}^{(f)}, x_{i+1}^{(f)}\right) .
$$


712 Generic uniqueness of minimal configurations

Assume that $y \in \mathcal{M}^{\mathrm{per}}\left(f_{\gamma}, \alpha\right)$. Formulas (4.1), (6.1), (6.2), and (6.4) imply that

$$
\begin{gathered}
\sum_{i=0}^{q-1} f\left(y_{i}, y_{i+1}\right)+\gamma \sum_{i=0}^{q-1} \phi_{f}\left(P_{1}\left(y_{i}\right)\right) \\
=\sum_{i=0}^{q-1} f_{\gamma}\left(y_{i}, y_{i+1}\right)=E_{\alpha}\left(f_{\gamma}\right)=E_{\alpha}(f) \leq \sum_{i=0}^{q-1} f\left(y_{i}, y_{i+1}\right), \\
\sum_{i=0}^{q-1} f\left(y_{i}, y_{i+1}\right)=\sum_{i=0}^{q-1} f_{\gamma}\left(y_{i}, y_{i+1}\right)=E_{\alpha}\left(f_{\gamma}\right)=E_{\alpha}(f), \\
y \in \mathcal{M}^{\text {per }}\left(f_{\gamma}, \alpha\right), \quad P_{1}\left(y_{i}\right) \in\left\{P_{1}\left(x_{j}^{(f)}\right): j=0, \ldots, q-1\right\}, \quad i=0, \ldots, q-1 .
\end{gathered}
$$

Since the set $\mathcal{M}^{\mathrm{per}}\left(f_{\gamma}, \alpha\right)$ is totally ordered, we conclude that $y$ is a translation of $x^{(f)}$. Thus

$$
\mathcal{M}^{\text {per }}\left(f_{\gamma}, \alpha\right)=\left\{T_{n} x^{(f)}: n=\left(n_{1}, n_{2}\right) \in \mathbb{Z}^{2}\right\} .
$$

By Proposition 4.8 and (6.6), there exists

$$
x^{\left(f^{+}\right)} \in \mathcal{M}^{\mathrm{per}}\left(f_{\gamma}, \alpha\right)
$$

such that

$$
x^{(f)}<x^{\left(f^{+}\right)}, \quad\left\{z \in \mathcal{M}^{\text {per }}\left(f_{\gamma}, \alpha\right): x^{(f)}<z<x^{\left(f^{+}\right)}\right\}=\varnothing .
$$

Proposition 1.7 implies that there exists

$$
y^{(f \gamma)} \in \mathcal{M}^{+}\left(f_{\gamma}, \alpha\right)
$$

such that

$$
\begin{gathered}
x^{(f)}<y^{(f \gamma)}<x^{\left(f^{+}\right)}, \\
\lim _{i \rightarrow \infty} y_{i}^{(f \gamma)}-x_{i}^{\left(f^{+}\right)}=0, \quad \lim _{i \rightarrow-\infty} y_{i}^{(f \gamma)}-x_{i}^{(f)}=0 .
\end{gathered}
$$

Define

$$
\Omega=\left\{P_{1}\left(y_{i}^{(f \gamma)}\right): i \in \mathbb{Z}\right\} \cup\left\{P_{1}\left(x_{i}^{(f)}\right): i \in \mathbb{Z}\right\}
$$

It is easy to see that $\Omega$ is a closed subset of $\mathbb{R}^{1} / \mathbb{Z}$.

By Corollary 4.2 , there exists a nonnegative function $\psi_{f \gamma} \in \mathbb{C}^{\infty}\left(\mathbb{R}^{1} / \mathbb{Z}\right)$ such that

$$
\left\{z \in \mathbb{R}^{1} / \mathbb{Z}: \psi_{f \gamma}(z)=0\right\}=\Omega .
$$

Let $\mu \in(0,1)$. Define $f_{\gamma \mu}: \mathbb{R}^{2} \rightarrow \mathbb{R}^{1}$ by

$$
f_{\gamma \mu}\left(\xi_{1}, \xi_{2}\right)=f_{\gamma}\left(\xi_{1}, \xi_{2}\right)+\mu \psi_{f \gamma}\left(P_{1}\left(\xi_{1}\right)\right), \quad\left(\xi_{1}, \xi_{2}\right) \in \mathbb{R}^{2} .
$$


It is easy to see that $f_{\gamma \mu} \in \mathfrak{M}_{k 0}$. Formulas (4.1), (6.4), (6.12), (6.13), and (6.14) imply that

$$
\begin{aligned}
E_{\alpha}\left(f_{\gamma}\right) & \leq E_{\alpha}\left(f_{\gamma \mu}\right) \leq \sum_{i=0}^{q-1} f_{\gamma \mu}\left(x_{i}^{(f)}, x_{i+1}^{(f)}\right) \\
& =\sum_{i=0}^{q-1} f_{\gamma}\left(x_{i}^{(f)}, x_{i+1}^{(f)}\right)+\mu \sum_{i=0}^{q-1} \psi_{f \gamma}\left(P_{1}\left(x_{i}^{(f)}\right)\right) \\
& =\sum_{i=0}^{q-1} f_{\gamma}\left(x_{i}^{(f)}, x_{i+1}^{(f)}\right)=E_{\alpha}(f)=E_{\alpha}\left(f_{\gamma}\right), \\
E_{\alpha}\left(f_{\gamma \mu}\right)= & E_{\alpha}\left(f_{\gamma}\right)=E_{\alpha}(f)=\sum_{i=0}^{q-1} f_{\gamma \mu}\left(x_{i}^{(f)}, x_{i+1}^{(f)}\right) \\
= & \sum_{i=0}^{q-1} f_{\gamma}\left(x_{i}^{(f)}, x_{i+1}^{(f)}\right)=\sum_{i=0}^{q-1} f\left(x_{i}^{(f)}, x_{i+1}^{(f)}\right) .
\end{aligned}
$$

Assume that

$$
y \in M^{\mathrm{per}}\left(f_{\gamma \mu}, \alpha\right)
$$

By (4.1), (6.14), (6.16), and (6.17),

$$
\begin{gathered}
\sum_{i=0}^{q-1} f_{\gamma}\left(y_{i}, y_{i+1}\right)+\sum_{i=0}^{q-1} \mu \psi_{f \gamma}\left(P_{1}\left(y_{i}\right)\right) \\
=\sum_{i=0}^{q-1} f_{\gamma \mu}\left(y_{i}, y_{i+1}\right)=E_{\alpha}\left(f_{\gamma \mu}\right)=E_{\alpha}\left(f_{\gamma}\right) \leq \sum_{i=0}^{q-1} f_{\gamma}\left(y_{i}, y_{i+1}\right), \\
\sum_{i=0}^{q-1} f_{\gamma}\left(y_{i}, y_{i+1}\right)=E_{\alpha}\left(f_{\gamma}\right),
\end{gathered}
$$

and $y \in M^{\text {per }}\left(f_{\gamma}, \alpha\right)$. Now, (6.6) implies that $y$ is a translation of $x^{(f)}$. Thus

$$
\mathcal{M}^{\text {per }}\left(f_{\gamma \mu}, \alpha\right)=\left\{T_{n} x^{(f)}: n=\left(n_{1}, n_{2}\right) \in \mathbb{Z}^{2}\right\} .
$$

Lemma 6.1. Let $z \in \mathcal{M}^{+}\left(f_{\gamma \mu}, \alpha\right)$. Then there exists $m=\left(m_{1}, m_{2}\right) \in \mathbb{Z}^{2}$ such that $T_{m} y^{(f \gamma)}=z$. Proof. By (6.8), (6.19), Proposition 4.8, and the definition of $\mathcal{M}^{+}(h, \alpha)$ with $h$ satisfying (H1), (H2), (H3), and (H4) (see Section 1), we may assume without loss of generality that

$$
x^{(f)}<z<x^{\left(f^{+}\right)} .
$$

Then it follows from Propositions 1.7 and 1.8 , the definition of $\mathcal{M}^{+}(h, \alpha)$ with $h$ satisfying (H1), (H2), (H3), and (H4), and (6.8) that

$$
\lim _{i \rightarrow \infty} x_{i}^{\left(f^{+}\right)}-z_{i}=0, \quad \lim _{i \rightarrow-\infty} x_{i}^{(f)}-z_{i}=0 .
$$




\section{Generic uniqueness of minimal configurations}

Since the set $\mathcal{M}^{+}\left(f_{\gamma \mu}, \alpha\right)$ is totally ordered (see Proposition 1.8), in order to prove the lemma, it is sufficient to show that there exist $m=\left(m_{1}, m_{2}\right) \in \mathbb{Z}^{2}$ and $i \in \mathbb{Z}$ such that $z_{i}=\left(T_{m} y^{(f \gamma)}\right)_{i}$. Assume the contrary. Then

$$
\left\{P_{1} z_{i}: i \in \mathbb{Z}\right\} \cap\left\{P_{1} y_{i}^{(f \gamma)}: i \in \mathbb{Z}\right\}=\varnothing
$$

Since the set $\mathcal{M}^{+}\left(f_{\gamma \mu}, \alpha\right) \cup \mathcal{M}^{\mathrm{per}}\left(f_{\gamma \mu}, \alpha\right)$ is totally ordered (see Proposition 1.7),

$$
\left\{P_{1} z_{i}: i \in \mathbb{Z}\right\} \cap\left\{P_{1} x_{i}^{(f)}: i \in \mathbb{Z}\right\}=\varnothing
$$

Formulas (6.12), (6.22), and (6.23) imply that

$$
\left\{P_{1} z_{i}: i \in \mathbb{Z}\right\} \cap \Omega=\varnothing \text {. }
$$

Formulas (6.13) and (6.24) imply that

$$
\psi_{f \gamma}\left(P_{1} z_{i}\right)>0 \quad \forall i \in \mathbb{Z}
$$

Choose a positive number

$$
\Delta<8^{-1} \mu \sum_{i=-q}^{q} \psi_{f \gamma}\left(P_{1} z_{i}\right) .
$$

By Proposition 1.4,

$$
\begin{gathered}
\left|z_{i}-z_{0}-i \alpha\right|<1 \quad \forall i \in \mathbb{Z}, \\
\left|y_{i}^{(f \gamma)}-y_{0}^{(f \gamma)}-i \alpha\right|<1 \quad \forall i \in \mathbb{Z} .
\end{gathered}
$$

Since the functions $f_{\gamma}$ and $f_{\gamma \mu}$ are continuous and periodic, there exists a number $\epsilon \in$ $(0,1)$ such that for each $\xi_{1}, \xi_{2}, \xi_{3}, \xi_{4} \in \mathbb{R}^{1}$ satisfying

$$
\begin{gathered}
\left|\xi_{1}-\xi_{2}\right|,\left|\xi_{3}-\xi_{4}\right| \leq 2|\alpha|+8, \\
\left|\xi_{1}-\xi_{3}\right| \leq 2 \epsilon, \quad\left|\xi_{2}-\xi_{4}\right| \leq 2 \epsilon
\end{gathered}
$$

the following inequality holds:

$$
\left|h\left(\xi_{1}, \xi_{2}\right)-h\left(\xi_{3}, \xi_{4}\right)\right| \leq \frac{\Delta}{16}, \quad h \in\left\{f_{\gamma}, f_{\gamma \mu}\right\}
$$

It follows from (6.11) and (6.21) that there exists an integer $m_{0}>4+4 q$ such that

$$
\left|z_{i}-y_{i}^{(f \gamma)}\right|<\frac{\epsilon}{2}
$$


for all integers $i$ satisfying $|i| \geq m_{0}$. Define $u \in \mathbb{R}^{\mathbb{Z}}$ as follows:

$$
\begin{gathered}
u_{i}=z_{i}, \quad i \in\left[\left(-\infty,-m_{0}-1\right] \cup\left[m_{0}+1, \infty\right)\right] \cap \mathbb{Z}, \\
u_{i}=y_{i}^{(f \gamma)}, \quad i \in\left[-m_{0}, m_{0}\right] \cap \mathbb{Z}
\end{gathered}
$$

We will show that

$$
\sum_{i=-m_{0}-1}^{m_{0}} f_{\gamma \mu}\left(z_{i}, z_{i+1}\right)-\sum_{i=-m_{0}-1}^{m_{0}} f_{\gamma \mu}\left(u_{i}, u_{i+1}\right)>0 .
$$

It follows from (6.32) that

$$
\begin{aligned}
& \sum_{i=-m_{0}-1}^{m_{0}} f_{\gamma \mu}\left(z_{i}, z_{i+1}\right)-\sum_{i=-m_{0}-1}^{m_{0}} f_{\gamma \mu}\left(u_{i}, u_{i+1}\right) \\
& =\sum_{i=-m_{0}-1}^{m_{0}} f_{\gamma \mu}\left(z_{i}, z_{i+1}\right)-f_{\gamma \mu}\left(z_{-m_{0}-1}, y_{-m_{0}}^{(f \gamma)}\right) \\
& \quad-f_{\gamma \mu}\left(y_{m_{0}}^{(f \gamma)}, z_{m_{0}+1}\right)-\sum_{i=-m_{0}}^{m_{0}-1} f_{\gamma \mu}\left(y_{i}^{(f \gamma)}, y_{i+1}^{(f \gamma)}\right) .
\end{aligned}
$$

By the definition of $\epsilon$ (see (6.29) and (6.30)), (6.27), (6.28), and (6.31),

$$
\begin{aligned}
& \mid f_{\gamma \mu}\left(z_{-m_{0}-1}, z_{-m_{0}}\right)+f_{\gamma \mu}\left(z_{m_{0}}, z_{m_{0}+1}\right) \\
& \quad-f_{\gamma \mu}\left(z_{-m_{0}-1}, y_{-m_{0}}^{(f \gamma)}\right)-f_{\gamma \mu}\left(y_{m_{0}}^{(f \gamma)}, z_{m_{0}+1}\right) \mid \leq \frac{\Delta}{8} .
\end{aligned}
$$

This inequality, (6.12), (6.13), (6.14), (6.26), and (6.34) imply that

$$
\begin{aligned}
& \sum_{i=-m_{0}-1}^{m_{0}} f_{\gamma \mu}\left(z_{i}, z_{i+1}\right)-\sum_{i=-m_{0}-1}^{m_{0}} f_{\gamma \mu}\left(u_{i}, u_{i+1}\right) \\
& \quad \geq \sum_{i=-m_{0}}^{m_{0}-1} f_{\gamma \mu}\left(z_{i}, z_{i+1}\right)-\sum_{i=-m_{0}}^{m_{0}-1} f_{\gamma \mu}\left(y_{i}^{(f \gamma)}, y_{i+1}^{(f \gamma)}\right)-8^{-1} \Delta \\
& \quad=-8^{-1} \Delta+\sum_{i=-m_{0}}^{m_{0}-1} f_{\gamma}\left(z_{i}, z_{i+1}\right)+\mu \sum_{i=-m_{0}}^{m_{0}-1} \psi_{f \gamma}\left(P_{1} z_{i}\right)-\sum_{i=-m_{0}}^{m_{0}-1} f_{\gamma}\left(y_{i}^{(f \gamma)}, y_{i+1}^{(f \gamma)}\right) \\
& >7 \Delta+\sum_{i=-m_{0}}^{m_{0}-1} f_{\gamma}\left(z_{i}, z_{i+1}\right)-\sum_{i=-m_{0}}^{m_{0}-1} f_{\gamma}\left(y_{i}^{(f \gamma)}, y_{i+1}^{(f \gamma)}\right) .
\end{aligned}
$$

Define

$$
v_{i}=z_{i}, \quad i=-m_{0}, \ldots, m_{0}, \quad v_{-m_{0}-1}=y_{-m_{0}-1}^{(f \gamma)}, \quad v_{m_{0}+1}=y_{m_{0}+1}^{(f \gamma)} .
$$


Since $y^{(f \gamma)} \in M\left(f_{\gamma}, \alpha\right)$, it follows from (6.28), the definition of $\epsilon$ (see (6.29) and (6.30)), and (6.31) that

$$
\begin{aligned}
0 \leq & \sum_{i=-m_{0}-1}^{m_{0}} f_{\gamma}\left(v_{i}, v_{i+1}\right)-\sum_{i=-m_{0}-1}^{m_{0}} f_{\gamma}\left(y_{i}^{(f \gamma)}, y_{i+1}^{(f \gamma)}\right) \\
\leq & \sum_{i=-m_{0}}^{m_{0}-1} f_{\gamma}\left(z_{i}, z_{i+1}\right)-\sum_{i=-m_{0}}^{m_{0}-1} f_{\gamma}\left(y_{i}^{(f \gamma)}, y_{i+1}^{(f \gamma)}\right)+f_{\gamma}\left(y_{-m_{0}-1}^{(f \gamma)}, z_{-m_{0}}\right) \\
& +f_{\gamma}\left(z_{m_{0}}, y_{m_{0}+1}^{(f \gamma)}\right)-f_{\gamma}\left(y_{-m_{0}-1}^{(f \gamma)}, y_{-m_{0}}^{(f \gamma)}\right)-f_{\gamma}\left(y_{m_{0}}^{(f \gamma)}, y_{m_{0}+1}^{(f \gamma)}\right) \\
\leq & \sum_{i=-m_{0}}^{m_{0}-1} f_{\gamma}\left(z_{i}, z_{i+1}\right)-\sum_{i=-m_{0}}^{m_{0}-1} f_{\gamma}\left(y_{i}^{(f \gamma)}, y_{i+1}^{(f \gamma)}\right)+\frac{\Delta}{8} .
\end{aligned}
$$

By these inequalities and (6.36),

$$
\sum_{i=-m_{0}-1}^{m_{0}} f_{\gamma \mu}\left(z_{i}, z_{i+1}\right)-\sum_{i=-m_{0}-1}^{m_{0}} f_{\gamma \mu}\left(u_{i}, u_{i+1}\right)>7 \Delta+\left(\frac{-\Delta}{8}\right)>6 \Delta,
$$

a contradiction. The contradiction we have reached proves Lemma 6.1.

Completion of the proof of Proposition 3.3. By Theorem 2.2, there exists a set $\mathscr{F}_{0} \subset \mathfrak{M}_{k 0}$ which is a countable intersection of open (in the weak topology) everywhere dense (in the strong topology) subsets of $\mathfrak{M}_{k}$. It is easy to see that for each $f \in \mathfrak{M}_{k 0}, \lim _{\gamma \rightarrow 0^{+}} f_{\gamma}=f$ in the strong topology, and that for each $f \in \mathfrak{M}_{k 0}$ and each $\gamma \in(0,1), \lim _{\mu \rightarrow 0^{+}} f_{\gamma \mu}=f_{\gamma}$ in the strong topology. Therefore, the set

$$
\mathscr{D}:=\left\{f_{\gamma \mu}: f \in \mathfrak{M}_{k 0}, \gamma, \mu \in(0,1)\right\}
$$

is an everywhere dense subset of $\mathfrak{M}_{k}$ with the strong topology.

Let $g \in \mathscr{D}$. By (6.19), (6.40), Propositions 1.7 and 4.8, and Lemma 6.1, there exist $x^{(g)}, x^{\left(g^{+}\right)} \in M^{\text {per }}(g, \alpha)$ and $y^{(g)} \in M^{+}(g, \alpha)$ such that

$$
\begin{gathered}
\mathcal{M}^{\text {per }}(g, \alpha)=\left\{T_{n} x^{(g)}: n=\left(n_{1}, n_{2}\right) \in \mathbb{Z}^{2}\right\}, \\
\mathcal{M}^{+}(g, \alpha)=\left\{T_{n} y^{(g)}: n=\left(n_{1}, n_{2}\right) \in \mathbb{Z}^{2}\right\}, \\
x^{(g)}<y^{(g)}<x^{\left(g^{+}\right)}, \quad\left\{z \in \mathcal{M}^{\text {per }}(g, \alpha): x^{(g)}<z<x^{\left(g^{+}\right)}\right\}=\varnothing .
\end{gathered}
$$

Let $j \geq 1$ be an integer. By Proposition 4.10 and Lemma 5.6, there is an open neighborhood $U(g, j)$ of $g$ in $\mathfrak{M}_{k}$ with the weak topology such that the following properties hold:

(a) for each $f \in \mathcal{U}(g, j) \cap \mathfrak{M}_{k 0}$ and each $x \in \mathcal{M}^{\text {per }}(f, \alpha)$, there exists $m=\left(m_{1}, m_{2}\right) \in$ $\mathbb{Z}^{2}$ such that $\left|x_{i}-\left(T_{m} x^{(g)}\right)_{i}\right|<(2 j)^{-1}$ for all $i \in \mathbb{Z}$;

(b) for each $f \in \mathcal{U}(g, j) \cap \mathfrak{M}_{k 0}$ and each $y \in \mathcal{M}^{+}(f, \alpha)$, there exists $m=\left(m_{1}, m_{2}\right) \in$ $\mathbb{Z}^{2}$ such that $\left|\left(T_{m} y\right)_{i}-x_{i}^{(g)}\right|<(2 j)^{-1}$ for all $i \in \mathbb{Z}$ or $\left|\left(T_{m} y\right)_{i}-y_{i}^{(g)}\right|<(2 j)^{-1}$ for all $i \in \mathbb{Z}$.

Define

$$
\mathscr{F}_{\alpha^{+}}=\mathscr{F}_{0} \cap\left[\cap_{n=1}^{\infty} \cup\{U(g, j): g \in \mathscr{D}, j \geq n\}\right] .
$$


It is not difficult to see that $\mathscr{F}_{\alpha^{+}}$is a countable intersection of open (in the weak topology) everywhere dense (in the strong topology) subsets of $\mathfrak{M}_{k}$.

Let $f \in \mathscr{F}_{\alpha^{+}}$. For each integer $n \geq 1$, there exist an integer $s_{n} \geq n$ and $g_{n} \in \mathscr{D}$ such that

$$
f \in \mathcal{U}\left(g_{n}, s_{n}\right) .
$$

Let $x, y \in \mathcal{M}^{\mathrm{per}}(f, \alpha)$. We will show that $y$ is a translation of $x$. It follows from property (a) and (6.45) that for each integer $n \geq 1$, there exists $m^{(n)}=\left(m_{1}^{(n)}, m_{2}^{(n)}\right) \in \mathbb{Z}^{2}$ such that

$$
\left|y_{i}-\left(T_{m^{(n)}} x\right)_{i}\right|<s_{n}^{-1} \leq \frac{1}{n} \quad \forall i \in \mathbb{Z} .
$$

By the periodicity of $y$ and $x$, we may assume without loss of generality that

$$
m_{1}^{(n)} \in[0, q] \quad \forall n \geq 1 .
$$

Then (6.46) implies that the sequence $\left\{m_{2}^{(n)}\right\}_{n=1}^{\infty}$ is bounded. By extracting a subsequence, we may assume without loss of generality that

$$
m^{(n)}=m^{(1)}, \quad n=1,2, \ldots
$$

Then (6.46) implies that for all integers $n \geq 1$,

$$
\left|y_{i}-\left(T_{m^{(1)}} x\right)_{i}\right|<\frac{1}{n}, \quad i \in \mathbb{Z} .
$$

Therefore, $y=T_{m_{1}} x$. Fix $\bar{x} \in \mathcal{M}^{\mathrm{per}}(f, \alpha)$. We have shown that

$$
\mathcal{M}^{\mathrm{per}}(f, \alpha)=\left\{T_{n} \bar{x}: n=\left(n_{1}, n_{2}\right) \in \mathbb{Z}^{2}\right\} .
$$

Proposition 4.8 implies that there exists

$$
\bar{x}^{+} \in \mathcal{M}^{\text {per }}(f, \alpha)
$$

such that

$$
\bar{x}<\bar{x}^{+}, \quad\left\{z \in \mathcal{M}^{\text {per }}(f, \alpha): \bar{x}<z<\bar{x}^{+}\right\}=\varnothing .
$$

By (6.51), (6.52), and Proposition 1.7, there exists $y^{(0)} \in \mathcal{M}^{+}(f, \alpha)$ such that

$$
\bar{x}<y^{(0)}<\bar{x}^{+} .
$$

Assume that $y \in M^{+}(f, \alpha)$. We will show that $y$ is a translation of $y^{(0)}$. By the definition of $\mathcal{M}^{+}(f, \alpha)$, Proposition 4.8, and (6.50), we may assume without loss of generality that

$$
\bar{x}<y<\bar{x}^{+} .
$$

By (6.45) and property (b) for each integer $n \geq 1$, there exist $r^{(n)}=\left(r_{1}^{(n)}, r_{2}^{(n)}\right) \in \mathbb{Z}^{2}$ and $l^{(n)}=\left(l_{1}^{(n)}, l_{2}^{(n)}\right) \in \mathbb{Z}^{2}$ such that

$$
\left|y_{i}^{(0)}-\left(T_{r^{(n)}} y^{\left(g_{n}\right)}\right)_{i}\right|<\left(2 s_{n}\right)^{-1} \leq(2 n)^{-1} \quad \forall i \in \mathbb{Z},
$$


or

$$
\begin{gathered}
\left|y_{i}^{(0)}-\left(T_{r^{(n)}} x^{\left(g_{n}\right)}\right)_{i}\right|<\left(2 s_{n}\right)^{-1} \leq(2 n)^{-1} \quad \forall i \in \mathbb{Z}, \\
\left|y_{i}-\left(T_{l^{(n)}} y^{\left(g_{n}\right)}\right)_{i}\right|<\left(2 s_{n}\right)^{-1} \leq(2 n)^{-1} \quad \forall i \in \mathbb{Z},
\end{gathered}
$$

or

$$
\left|y_{i}-\left(T_{l^{(n)}} x^{\left(g_{n}\right)}\right)_{i}\right|<\left(2 s_{n}\right)^{-1} \leq(2 n)^{-1} \quad \forall i \in \mathbb{Z}
$$

Define

$$
E=\{n \in \mathbb{Z}: n \geq 1 \text { and (6.58) holds }\}
$$

Assume that the set $E$ is infinite. By the periodicity of $x^{\left(g_{n}\right)}, n \geq 1$, we may assume without loss of generality that

$$
l_{1}^{(n)} \in[0, q], \quad n \in E .
$$

Recall that $\left|x_{0}^{\left(g_{n}\right)}\right| \leq 1, n=1,2, \ldots$ Together with Proposition 1.4, this implies that for each $i \in \mathbb{Z}$,

$$
\left|x_{i}^{\left(g_{n}\right)}\right| \leq\left|x_{0}^{\left(g_{n}\right)}\right|+|i||\alpha|+1, \quad n=1,2, \ldots
$$

It follows from (6.58), (6.59), (6.60), and (6.61) that the set $\left\{l_{2}^{(n)}: n \in E\right\}$ is bounded. Therefore, the set $\left\{l^{(n)}: n \in E\right\}$ is bounded. There exists an infinite set $F \subset E$ such that $l^{\left(n_{1}\right)}=l^{\left(n_{2}\right)}$ for each $n^{(1)}, n^{(2)} \in F$. Combined with (6.58) and (6.59), this fact implies that $\left|\left(T_{l} y\right)_{i}-x_{i}^{\left(g_{n}\right)}\right|<(2 n)^{-1}$ for all $i \in \mathbb{Z}$ and all $n \in F$ with some $l \in \mathbb{Z}^{2}$. This implies that $y \in \mathcal{M}^{\text {per }}(f, \alpha)$, a contradiction. Therefore, $E$ is finite. Since $y$ is an arbitrary element of $\mathcal{M}^{+}(f, \alpha)$, the set

$$
\{n \in \mathbb{Z}: n \geq 1 \text { and (6.56) holds }\}
$$

is finite. We may assume without loss of generality that (6.55) and (6.57) hold for any integer $n \geq 1$. This fact implies that for each integer $n \geq 1$, there exists $j^{(n)}=\left(j_{1}^{(n)}, j_{2}^{(n)}\right) \in$ $\mathbb{Z}^{2}$ such that

$$
\left|y_{i}-\left(T_{j(n)} y^{(0)}\right)_{i}\right|<\frac{1}{n} \quad \forall i \in \mathbb{Z}
$$

It follows from (6.52), (6.53), (6.54), (6.63), and the definition of $M^{+}(f, \alpha)$ that

$$
\begin{array}{ll}
\lim _{i \rightarrow-\infty} \bar{x}_{i}-y_{i}^{(0)}=0, & \lim _{i \rightarrow-\infty} \bar{x}_{i}-y_{i}=0, \\
\lim _{i \rightarrow \infty} \bar{x}_{i}^{+}-y_{i}^{(0)}=0, & \lim _{i \rightarrow \infty} \bar{x}_{i}^{+}-y_{i}=0 .
\end{array}
$$


Formulas (6.63) and (6.64) imply that for each integer $n \geq 1$,

$$
\begin{aligned}
& \lim _{i \rightarrow \infty}\left[\left(T_{j^{(n)}} \bar{x}^{+}\right)_{i}-\left(T_{j^{(n)}} y^{(0)}\right)_{i}\right]=0, \\
& \limsup _{i \rightarrow \infty}\left[\left|\bar{x}_{i}^{+}-\left(T_{j^{(n)} \bar{x}^{+}}\right)_{i}\right|\right] \leq \lim _{i \rightarrow \infty}\left|\bar{x}_{i}^{+}-y_{i}\right|+\limsup _{i \rightarrow \infty}\left[\left|y_{i}-\left(T_{j^{(n)}} y^{(0)}\right)_{i}\right|\right] \\
& +\lim _{i \rightarrow \infty}\left|\left[\left(T_{j^{(n)}} y^{(0)}\right)_{i}-\left(T_{j^{(n)} \bar{x}^{+}}\right)_{i}\right]\right| \leq \frac{1}{n} .
\end{aligned}
$$

Since $\bar{x}^{+}$is periodic, we obtain that for any integer $n \geq 1$,

$$
\left|\bar{x}_{i}^{+}-\left(T_{j^{(n)}} \bar{x}^{+}\right)_{i}\right| \leq \frac{1}{n}, \quad i \in \mathbb{Z}
$$

By Corollary 4.4 and (6.50), there exists $\kappa \in(0,1)$ such that for each $z^{(1)}, z^{(2)} \in \mathcal{M}^{\text {per }}(f, \alpha)$ satisfying $z^{(1)} \neq z^{(2)}$,

$$
\left|z_{i}^{(1)}-z_{i}^{(2)}\right|>2 \kappa, \quad i \in \mathbb{Z}
$$

Formulas (6.66) and (6.67) imply that for any integer $n>2 \kappa^{-1}$,

$$
\bar{x}^{+}=T_{j^{(n)}} \bar{x}^{+}, \quad \bar{x}_{i}^{+}=\bar{x}_{i-j_{1}^{(n)}}^{+}+j_{2}^{(n)} \quad \forall i \in \mathbb{Z},
$$

and that the rotation number $\alpha$ of $\bar{x}^{+}$satisfies $\alpha=p / q=j_{2}^{(n)} / j_{1}^{(n)}$. Since $p / q$ is an irreducible fraction, we obtain that for any integer $n>2 \kappa^{-1}$, there is an integer $a_{n}$ such that

$$
a_{n}(p, q)=j^{(n)}
$$

We have three cases:

(1) there exists a strictly increasing sequence of natural numbers $\left\{n_{t}\right\}_{t=1}^{\infty}$ such that $\lim _{t \rightarrow \infty} a_{n_{t}}=\infty$;

(2) there exists a strictly increasing sequence of natural numbers $\left\{n_{t}\right\}_{t=1}^{\infty}$ such that $\lim _{t \rightarrow \infty} a_{n_{t}}=-\infty$;

(3) there exists a strictly increasing sequence of natural numbers $\left\{n_{t}\right\}_{t=1}^{\infty}$ such that $a_{n_{t}}=a_{n_{1}}$ for all integers $t \geq 1$.

Assume that case (1) holds. Then, by (6.50), (6.64), and (6.69) for any integer $i$,

$$
\begin{aligned}
\left(T_{j^{\left(n_{t}\right)}} y^{(0)}\right)_{i} & =\left(T_{a_{n_{t}}(q, p)} y^{(0)}\right)_{i}=y_{i-a_{n_{t}} q}^{(0)}+a_{n_{t}} p, \\
\left(T_{j^{\left(n_{t}\right)}} y^{(0)}\right)_{i}-\bar{x}_{i} & =y_{i-a_{n_{t}} q}^{(0)}+a_{n_{t}} p-\left(\bar{x}_{i-a_{n_{t}} q}+a_{n_{t}} p\right) \\
& =y_{i-a_{n_{t}} q}^{(0)}-\bar{x}_{i-a_{n_{t}} q} \longrightarrow 0 \quad \text { as } t \longrightarrow \infty, \\
\left(T_{j^{\left(n_{t}\right)}} y^{(0)}\right)_{i}-\bar{x}_{i} & \longrightarrow 0 \text { as } t \longrightarrow \infty, \forall i \in \mathbb{Z} .
\end{aligned}
$$

This contradicts (6.63). Therefore, case (1) does not hold. 
Analogously, we can show that case (2) does not hold. Indeed, assume that case (2) holds. Then, by (6.50), (6.64), and (6.69) for any integer $i$,

$$
\begin{aligned}
\left(T_{\left.j^{\left(n_{t}\right.}\right)} y^{(0)}\right)_{i} & =\left(T_{a_{n_{t}}(q, p)} y^{(0)}\right)_{i}=y_{i-a_{n_{t}} q}^{(0)}+a_{n_{t}} p, \\
\left(T_{j^{\left(n_{t}\right)}} y^{(0)}\right)_{i}-\bar{x}_{i}^{+} & =y_{i-a_{n_{t}} q}^{(0)}+a_{n_{t}} p-\left(\bar{x}_{i-a_{n_{t}} q}^{+}+a_{n_{t}} p\right) \\
& =y_{i-a_{n_{t}} q}^{(0)}-\bar{x}_{i-a_{n_{t}} q}^{+} \longrightarrow 0 \text { as } t \longrightarrow \infty, \\
\left(T_{j^{\left(n_{t}\right)}} y^{(0)}\right)_{i}-\bar{x}_{i}^{+} & \longrightarrow 0 \text { as } t \longrightarrow \infty, \forall i \in \mathbb{Z} .
\end{aligned}
$$

This contradicts (6.63). Therefore, case (2) does not hold. We have shown that case (3) is valid. Then it follows from (6.63) and (6.69) that for all $i \in \mathbb{Z}$ and any integer $t \geq 1$,

$$
\frac{1}{n}>\left|y_{i}-\left(T_{a_{n_{t}}(q, p)} y^{(0)}\right)_{i}\right|=\left|y_{i}-\left(T_{a_{n_{1}}(q, p)} y^{(0)}\right)_{i}\right|
$$

and $y=T_{a_{n_{1}}(q, p)} y^{(0)}$. Proposition 3.3 is proved.

\section{References}

[1] S. Aubry, The twist map, the extended Frenkel-Kontorova model and the devil's staircase, Phys. D 7 (1983), no. 1-3, 240-258.

[2] S. Aubry and P. Y. Le Daeron, The discrete Frenkel-Kontorova model and its extensions. I. Exact results for the ground-states, Phys. D 8 (1983), no. 3, 381-422.

[3] V. Bangert, Mather sets for twist maps and geodesics on tori, Dynamics Reported, Vol. 1, Dynam. Report. Ser. Dynam. Systems Appl., vol. 1, Wiley, Chichester, 1988, pp. 1-56.

[4] Geodesic rays, Busemann functions and monotone twist maps, Calc. Var. Partial Differential Equations 2 (1994), no. 1, 49-63.

[5] M. L. Byalyı̆ and L. V. Polterovich, Geodesic flows on the two-dimensional torus and phase transitions "commensurability-incommensurability", Funct. Anal. Appl. 20 (1986), 260-266.

[6] W. E, Aubry-Mather theory and periodic solutions of the forced Burgers equation, Comm. Pure Appl. Math. 52 (1999), no. 7, 811-828.

[7] W. E, K. Khanin, A. Mazel, and Ya. Sinai, Invariant measures for Burgers equation with stochastic forcing, Ann. of Math. (2) 151 (2000), no. 3, 877-960.

[8] W. E and Ya. Sinai, Recent results on mathematical and statistical hydrodynamics, Russian Math. Surveys 55 (2000), 635-666.

[9] J. L. Kelley, General Topology, D. Van Nostrand, New York, 1955.

[10] A. Leizarowitz and V. J. Mizel, One-dimensional infinite-horizon variational problems arising in continuum mechanics, Arch. Rational Mech. Anal. 106 (1989), no. 2, 161-194.

[11] M. Marcus and A. J. Zaslavski, The structure and limiting behavior of locally optimal minimizers, Ann. Inst. H. Poincaré Anal. Non Linéaire 19 (2002), no. 3, 343-370.

[12] J. N. Mather, Existence of quasiperiodic orbits for twist homeomorphisms of the annulus, Topology 21 (1982), no. 4, 457-467.

[13] Minimal measures, Comment. Math. Helv. 64 (1989), no. 3, 375-394.

[14]_, Differentiability of the minimal average action as a function of the rotation number, Bol. Soc. Brasil. Mat. (N.S.) 21 (1990), no. 1, 59-70.

[15]__ Action minimizing invariant measures for positive definite Lagrangian systems, Math. Z. 207 (1991), no. 2, 169-207.

[16] J. Moser, Minimal solutions of variational problems on a torus, Ann. Inst. H. Poincaré Anal. Non Linéaire 3 (1986), no. 3, 229-272. 
[17] Recent developments in the theory of Hamiltonian systems, SIAM Rev. 28 (1986), no. 4, 459-485.

[18] Ya. Sinai, Topics in Ergodic Theory, Princeton Mathematical Series, vol. 44, Princeton University Press, New Jersey, 1994.

[19] A. J. Zaslavski, Ground states in Frenkel-Kontorova model, Math. USSR Izv. 29 (1987), 323-354.

[20] _ The existence and structure of extremals for a class of second order infinite horizon variational problems, J. Math. Anal. Appl. 194 (1995), no. 3, 660-696.

[21] Existence and structure of extremals for one-dimensional nonautonomous variational problems, J. Optim. Theory Appl. 97 (1998), no. 3, 731-757.

Alexander J. Zaslavski: Department of Mathematics, Technion - Israel Institute of Technology, 32000 Haifa, Israel

E-mail address: ajzasl@tx.technion.ac.il 


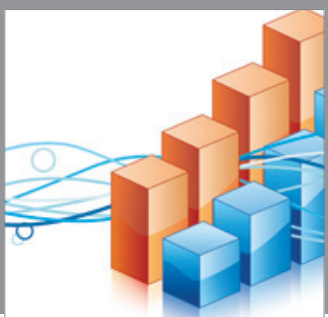

Advances in

Operations Research

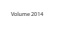

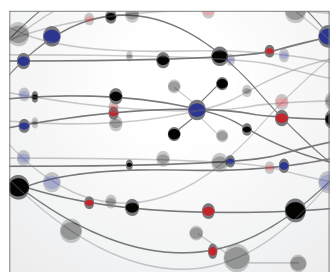

\section{The Scientific} World Journal
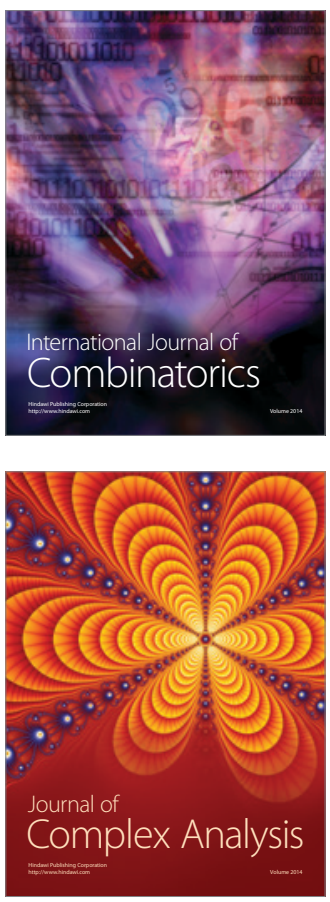

International Journal of

Mathematics and

Mathematical

Sciences
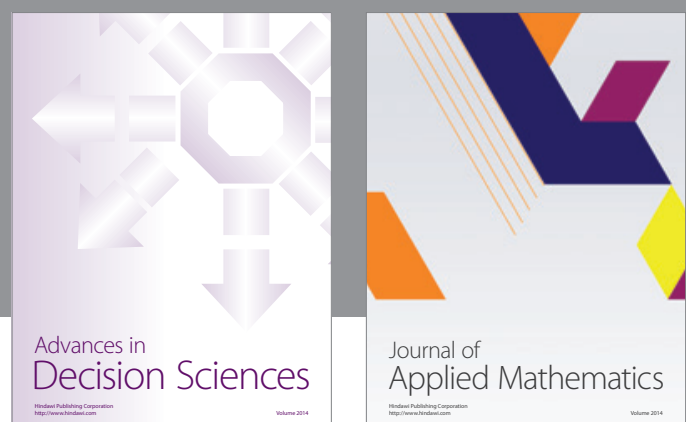

Journal of

Applied Mathematics
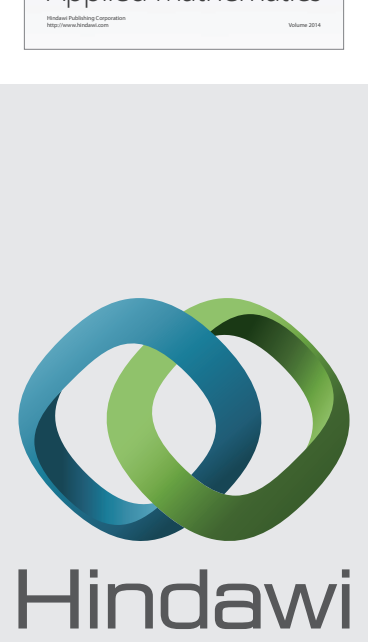

Submit your manuscripts at http://www.hindawi.com
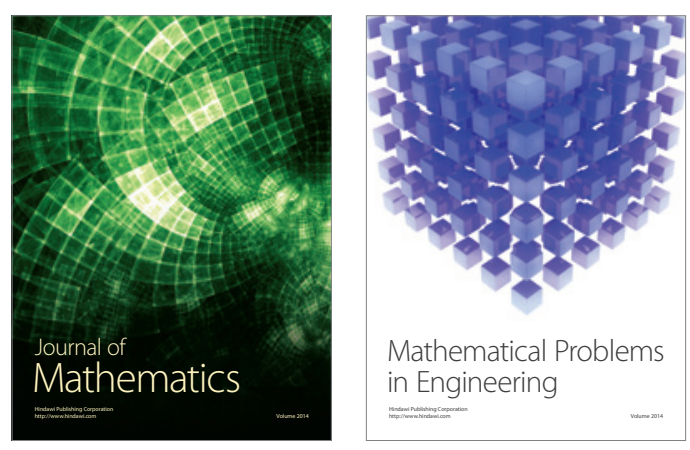

Mathematical Problems in Engineering
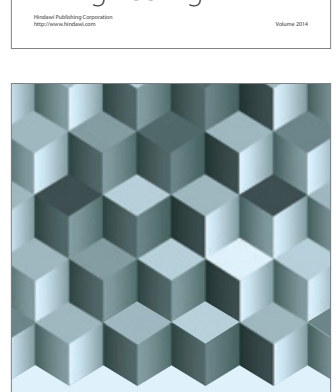

Journal of

Function Spaces
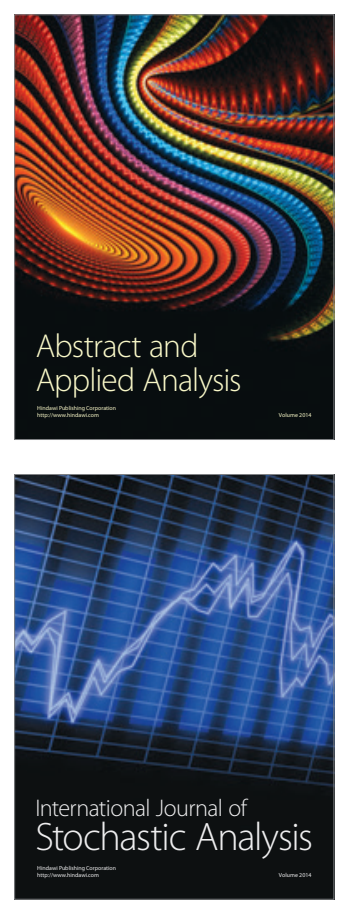

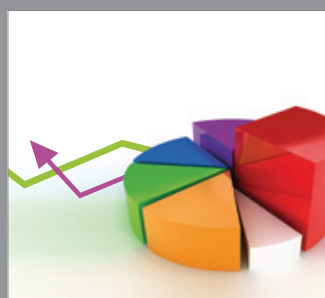

ournal of

Probability and Statistics

Promensencen
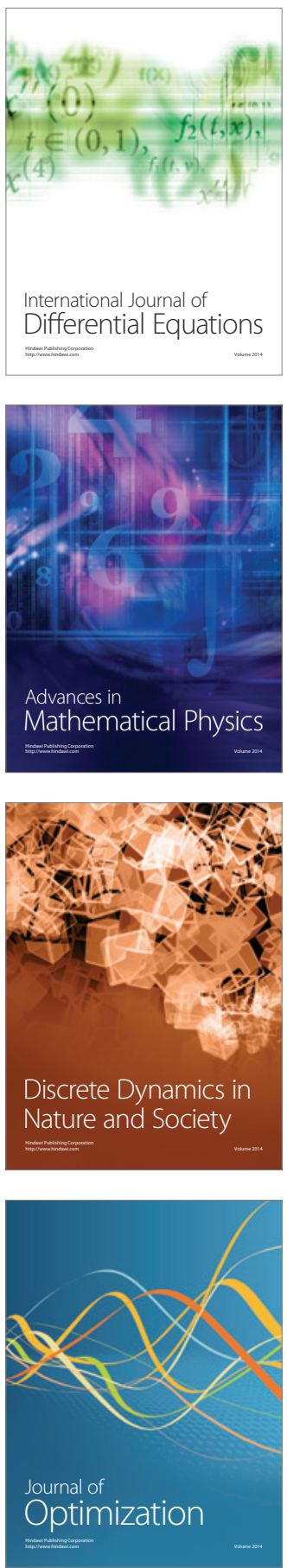